\title{
The effect of PD-L1 categories-directed pembrolizumab plus chemotherapy for newly diagnosed metastatic non-small-cell lung cancer: a cost-effectiveness analysis
}

\author{
Bin $\mathrm{Wu}^{1}$, Shun $\mathrm{Lu}^{2}$ \\ ${ }^{1}$ Medical Decision and Economic Group, Department of Pharmacy, Ren Ji Hospital, South Campus, School of Medicine, Shanghai Jiaotong \\ University, Shanghai, China; ${ }^{2}$ Shanghai Lung Cancer Center, Shanghai Chest Hospital Affiliated to Shanghai Jiaotong University, Shanghai, China \\ Contributions: (I) Conception and design: S Lu; (II) Administrative support: B Wu; (III) Provision of study materials or patients: S Lu; (IV) Collection \\ and assembly of data: B Wu; (V) Data analysis and interpretation: B Wu; (VI) Manuscript writing: All authors; (VII) Final approval of manuscript: All \\ authors. \\ Correspondence to: Prof. Shun Lu. Shanghai Lung Cancer Center, Shanghai Chest Hospital Affiliated to Shanghai Jiaotong University, 241 West \\ Huaihai Road, Shanghai, China. Email: lushunchest@163.com.
}

Background: The effectiveness of adding pembrolizumab to chemotherapy improve outcomes in newly diagnosed metastatic non-small-cell lung cancer (NSCLC). We aimed to evaluate the economic outcomes of first-line treatment by adding pembrolizumab to chemotherapy with and without the use of PD-L1 testing for patient selection.

Methods: A decision-analytic model was adopted to project the disease course of newly diagnosed metastatic nonsquamous and squamous NSCLC without EGFR or ALK mutations. The efficacy and toxicity data were gathered from the KEYNOTE-189 and KEYNOTE-407 trials. Transition probabilities were estimated from the reported survival probabilities in each group. Cost and health preference data were derived from published economic evaluations. The incremental cost-effectiveness ratio (ICER) was measured, and subgroup, one-way and probabilistic sensitivity analyses (PSA) were performed for exploring the model uncertainties.

Results: In the US context, pembrolizumab plus chemotherapy is projected to increase quality-adjustedlife year (QALY) by 1.168 and 0.988 in comparison with chemotherapy and the ICERs were \$122,248 and $\$ 121,375 /$ QALY in the whole nonsquamous and squamous patients with unconfirmed PD-L1 tumor proportion scores (TPS), respectively. After the selection of patients by PD-L1 TPS by PD-L1 testing, the ICERs of adding pembrolizumab treatment for patients with confirmed PD-L1 TPS $>1 \%$ and $\geq 50 \%$ were $\$ 143,282$ and $\$ 127,661$ /QALY in nonsquamous disease, and $\$ 131,495$ and $\$ 121,554$ QALY in squamous disease, respectively. The ICERs of adding pembrolizumab treatment for Chinese patients were higher than \$40,000/QALY regardless of the histology and TPS subgroups, which highly exceed the willingness-to-pay threshold of \$29,196/QALY (three times of per capita gross domestic product of China in 2018) in China.

Conclusions: Pembrolizumab plus chemotherapy as first-line treatment for untreated metastatic NSCLC without EGFR or ALK mutations is a cost-effective option regardless of PD-L1 expression status in the US context, and not cost-effective in the Chinese context. However, PD-L1 categories-directed pembrolizumab could not increase the cost-effectiveness of immunotherapy.

Keywords: Cost-effectiveness; programmed cell death 1 ligand 1 (PD-L1); pembrolizumab; metastatic non-smallcell lung cancer (NSCLC)

Submitted Nov 27, 2019. Accepted for publication Jul 17, 2020.

doi: $10.21037 /$ tlcr-19-605

View this article at: http://dx.doi.org/10.21037/tlcr-19-605 


\section{Introduction}

The Global Burden of Disease Study revealed that lung cancer is one of the leading causes of non-communicable disease burden worldwide (1). Approximately $85 \%$ to $90 \%$ of lung cancers are non-small-cell lung cancer (NSCLC), including $65 \%$ to $75 \%$ locally metastatic or metastatic disease. Platinum-based chemotherapy has been the standard of care for the first-line treatment of metastatic NSCLC that lacks sensitizing EGFR or ALK mutations (2). However, the overall survival (OS) and progressionfree survival (PFS) of chemotherapy are unsatisfied with metastatic NSCLC.

Recently, immune checkpoint inhibitors (ICIs) treatment that block the programmed cell death 1 ligand 1 (PD-L1) and programmed cell death 1 (PD-1) pathway has become to replace chemotherapy as the first-line regimen (3-5). The recent phase 3 KEYNOTE-189 and KEYNOTE-407 trials showed pembrolizumab plus chemotherapy provided longer OS and PFS in comparison with chemotherapy in patients metastatic NSCLC (6-8). Although the survival benefit associated with the pembrolizumab combination was observed in all subgroups of PD-L1 tumor proportion scores (TPS), the higher relative benefit was observed in the subgroup with a PD-L1 TPS of $1 \%$ to $49 \%$ and $\geq 50 \%$ than TPS of $1 \%$. This finding was coherent with the recent trials that showed the PDL-1-expression category could predict the benefit of ICIs treatment, although patients with PD-L1 negative tumors also benefited from its treatment as compared to chemotherapy (9). However, due to the high cost of implementing pembrolizumab in the first-line setting that limited the widely prescription in clinical practice, the cost-effectiveness of first-line pembrolizumab plus chemotherapy need to be evaluated. Furthermore, despite the promising activity of pembrolizumab plus chemotherapy for metastatic NSCLC irrespective of PD-L1 expression, the following unclear question also needs to be elucidated: will the patient selection based on PD-L1 expression lead to improving the cost-effectiveness of adding pembrolizumab to chemotherapy? Is there a potential cut-point value of PD-L1 expression for pembrolizumab plus chemotherapy that is cost-effective?

For increasing adoption of expensive agents by rationalizing therapy from a clinical standpoint thus making treatment more cost-effective, health policymakers and payers would assess the clinical value of the drug in different subgroups with varying responses to immunotherapy (10). The present evaluation investigates the economic outcomes of first-line pembrolizumab plus chemotherapy treatment with and without patient selection using varied $\mathrm{PD}$ L1 expression status in the US and Chinese context. We present the following article in accordance with the CHEERS Reporting Checklist (available at http://dx.doi. org/10.21037/tlcr-19-605).

\section{Methods}

\section{Analytic overview}

A mathematical model combining decision tree and Markov approach was established to measure the clinical and economic outcomes of adding pembrolizumab to chemotherapy for previously untreated metastatic nonsquamous and squamous NSCLC without EGFR or ALK mutations, which was similar with the KEYNOTE-189 and KEYNOTE-407 trials, respectively $(7,8)$. For the whole patients with unconfirmed PD-L1 TPS, the decision trees included four competing strategies (Figure 1A): chemotherapy for all patients (reference strategy); pembrolizumab plus chemotherapy for all patients without determination of PD-L1 status (universal pembrolizumab strategy); pembrolizumab plus chemotherapy for patients with PD-L1 TPS of $\geq 50 \%$ and chemotherapy for other patients after PD-L1 TPS was determined (TPS50 pembrolizumab strategy), and pembrolizumab plus chemotherapy for patients with PDL1 TPS of $\geq 1 \%$ and chemotherapy for other patients (TPS1 pembrolizumab strategy). For the three sub-populations with confirmed PD-L1 TPS $\geq 1 \%, 1 \%$ to $49 \%$ and $\geq 50 \%$, chemotherapy and pembrolizumab plus chemotherapy would be the competing strategies (Figure 1B). A threehealth-state Markov model was established to reflect the disease course, which included the following health states: progression-free disease (PFD), progressed disease (PD), and death. The Markov cycle length was 21-day in keeping with the treatment schedule reported by KEYNOTE-189 and KEYNOTE-407 trials $(7,8)$, and the time horizon was 20 years as previous studies done $(11,12)$. During each Markov cycle, the model redistributes the hypothetical patients among the three health states according to transition probabilities. The initial state is assumed to be PFD, and death is the absorbing state.

\section{Clinical data inputs}

PFS and OS for pembrolizumab plus chemotherapy and 
A A

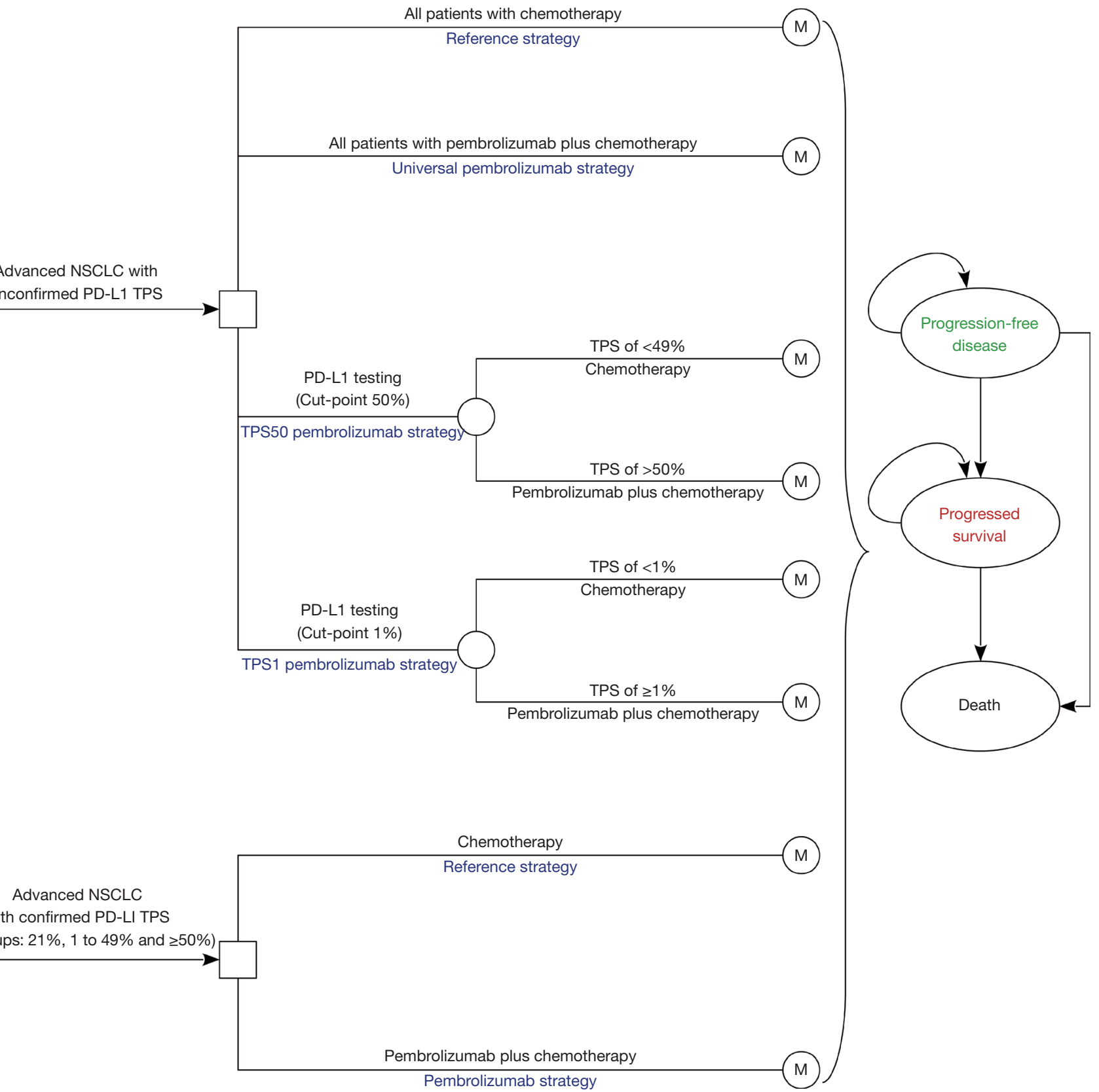

Figure 1 Model structure for previously untreated metastatic nonsquamous and squamous NSCLC without EGFR or ALK mutations. (A) The whole patients with unconfirmed PD-L1 TPS; (B) sub-populations with confirmed PD-L1 TPS $\geq 1 \%, 1 \%$ to $49 \%$ and $\geq 50 \%$. NSCLC, non-small-cell lung cancer; PD-L1, programmed cell death 1 ligand 1; TPS, tumor proportion score.

chemotherapy in metastatic nonsquamous and squamous NSCLC were informed by the results of KEYNOTE-189 and KEYNOTE-407 trials, respectively (7), which were extrapolated over the model time horizon using standard statistical analyses described by Guyot et al. (13). Graph Digitizer (version 2.26; http://getdata-graph-digitizer.com) was used to gather the data points from the PFS and OS curves, and these data points were then used to fit following parametric survival functions: Weibull, log-normal, loglogistic, exponential, generalized gamma, Gompertz and Royston/Parmar spline models (14). Goodness of fit was based on visual inspection and Akaike information criterion 
(AIC). The adopted model and AIC value for the whole intention-to-treat population and three subgroups of PD-L1 TPS (TPS of $<1 \%$, TPS of $1 \%$ to $49 \%$ and TPS of $\geq 50 \%$ ) were shown in Table S1. The modeled virtual patient-level data comprised event and censor times and were equal in number to the initial number at risk, which was closely reproduced the digitized Kaplan-Meier curves. The PFS and OS plots created by using the virtual patientlevel data and the predicted curves by using parametric survival models are shown in Figures S1-S4.

Due to the shorter follow-up time of KEYNOTE-189 and KEYNOTE-407 trials than the KEYNOTE-042 trial, we pooled the OS data of three trials for exploring the survival rates of chemotherapy to avoid the uncertainty around the long-term survival. Although the histology and chemotherapy regimen of these three trials were discrepant, our approach could be supported by the fact that no notable impact of histologies of advanced NSCLC and carboplatinversus cisplatin-based chemotherapy on OS $(2,15)$. By using the virtual patient-level data, we also compared the OS of three trials in the chemotherapy arm, and no notable difference was found (Figure S5). For the whole population receiving chemotherapy, Royston/Parmar spline model was adopted for extrapolating the pooled OS data, and the log-normal and log-logistic model for the PFS data from KEYNOTE-189 and KEYNOTE-407 trials, respectively. The PFS and OS probabilities of chemotherapy in three subgroups with confirmed PD-L1 TPS $<1 \%, 1 \%$ to $49 \%$ and $\geq 50 \%$ were calculated by multiplying the PFS and OS rates in the whole population receiving chemotherapy and the HRs of subgroups versus the whole population. The HRs were shown in Table S2, which were estimated by using the Cox proportional-hazards model after pooling the virtual patient-level data of the whole population and three subgroups receiving chemotherapy. The PFS and OS data for the whole population and three subgroups in pembrolizumab plus chemotherapy was estimated by multiplying the reported HRs and the PFS and OS rates in chemotherapy arms. The influences of HRs would be checked in sensitivity and subgroup analyses.

On the basis of the fitted PFS and OS model, denoted as $\mathrm{P}(\mathrm{t})$ and $\mathrm{S}(\mathrm{t})$, we computed the disease progression probability $\operatorname{Prob}_{(P F S \rightarrow P D)}$ and cancer-specific mortality $\operatorname{Prob}_{(P D \rightarrow \text { Deat })}$ at cycle $\mathrm{t}$ as: $\operatorname{Prob}_{(P F S \rightarrow P D)}=\left[\left(\mathrm{P}_{(\mathrm{t})}-\mathrm{P}_{(\mathrm{t}+1)}\right] / \mathrm{P}_{(\mathrm{t})}\right.$ and $\operatorname{Prob}_{(P D \rightarrow \text { Deat })}=\left[\left(\mathrm{S}_{(\mathrm{t})}-\mathrm{S}_{(\mathrm{t}+1)}\right] /\left[\left(\mathrm{S}_{(\mathrm{t})}-\mathrm{P}_{(\mathrm{t})}\right]\right.\right.$, respectively. After the disease progressed, the data of patients who received second-line active treatment was collected from the KEYNOTE-189 trial and KEYNOTE-407 trials, respectively $(7,8)$. The key clinic inputs were summarized in Table 1.

The model includes risks of all-cause adverse events (AEs) of grade $\geq 3$ reported by the KEYNOTE-189 and KEYNOTE-407 trials $(7,8)$. The impact of less severe or common AEs on model results would be expected to be negligible. The modeled probabilities of these AEs is described in Table 1.

\section{Cost and utility inputs}

Only direct medical costs were considered and stated in reported in 2018 US dollars, including the drug acquisition costs, costs attributed to the patient's health state, costs for the management of AEs, and costs of end-of-life care (Table 1). The costs associated with health care services were inflated to 2018 values according to the US consumer price index (17). In China, the costs were translated into 2018 US dollars $(\$ 1=$ CNY 6.8).

Based on the KEYNOTE-189 and KEYNOTE-407 trials, pembrolizumab was prescribed at a dose of $200 \mathrm{mg}$ on day 1 for up to 35 cycles with the combination of chemotherapy. The prices of pembrolizumab in the US (average wholesale price) were collected from public databases and the literature (18). In the US, the price of pembrolizumab was discounted at $17 \%$ to account for contract pricing (19). For the first 4 cycles, the cost related to cytotoxic chemotherapy for untreated metastatic NSCLC were $\$ 24,437$ per patient regardless of histology (20). For nonsquamous NSCLC, the cost related to maintenance chemotherapy was $\$ 5,887$ per cycle (20). After disease progressed, $44.6 \%$ in chemotherapy and $30.5 \%$ in pembrolizumab plus chemotherapy arm received subsequent active therapy (7). The average 21 day costs of disease management (excluding drug, drug administration, and AE related costs) in the PFD and PD states are stratified by years 1, 2, 3, 4 to 5 , and over 5 following first-line treatment initiation. The analysis included the costs related to managing grade $\geq 3$ AEs. The costs related to subsequent therapies taken following discontinuation of initial trial treatments, managing grade $\geq 3$ AEs, disease management and terminal care the last 30 days of life were extracted from literature $(11,12)$, which estimated the health resource utilization based on the KEYNOTE-189 and KEYNOTE-407 trials. In TPS1 and TPS50 pembrolizumab strategy, PD-L1 testing cost was considered (21). The cost estimates in the Chinese setting were showed in Table $S 3$. 
Table 1 Model parameters: baseline values, ranges, and distributions for sensitivity analysis

\begin{tabular}{|c|c|c|}
\hline Parameters & Nonsquamous non-small-cell lung cancer & Squamous non-small-cell lung cancer \\
\hline \multicolumn{3}{|l|}{ Survival model of chemotherapy } \\
\hline Model for PFS & $\begin{array}{l}\text { Log-normal, meanlog: } 1.9509 \text { (se: } 0.0681 \text { ), } \\
\text { sdlog: } 0.9444 \text { (se: 0.0537) }\end{array}$ & $\begin{array}{l}\text { Log-logistic, shape: } 1.872 \text { (se: } 0.112 \text { ), scale: } \\
7.053 \text { (se: } 0.402 \text { ) }\end{array}$ \\
\hline $\begin{array}{l}\text { HR of PFS of between pembrolizumab plus } \\
\text { chemotherapy and chemotherapy }\end{array}$ & $\begin{array}{l}0.48 \text { [range: } 0.4-0.58, \text { dist: lognormal } \\
(\text { log-mean }=-0.734, \text { log-sd }=3.081)]\end{array}$ & $\begin{array}{l}0.56 \text { [range: } 0.45-0.7, \text { dist: lognormal } \\
(\text { log-mean }=-0.58, \text { log-sd }=2.752)]\end{array}$ \\
\hline $\begin{array}{l}\text { HR of OS of between pembrolizumab plus } \\
\text { chemotherapy and chemotherapy }\end{array}$ & $\begin{array}{l}0.56 \text { [range: } 0.45-0.7, \text { dist: lognormal } \\
(\text { log-mean }=-0.58, \text { log-sd }=2.752)]\end{array}$ & $\begin{array}{l}0.64 \text { [range: } 0.49-0.85, \text { dist: lognormal } \\
(\text { log-mean }=-0.446, \text { log-sd }=2.388)]\end{array}$ \\
\hline $\begin{array}{l}\text { Grade } \geq 3 \text { AEs in pembrolizumab plus } \\
\text { chemotherapy treatment }\end{array}$ & $\begin{array}{l}0.672 \text { [range: } 0.504-0.84 \text {, dist: beta }(\alpha=5.2 \text {, } \\
\beta=2.6) \text { ] }\end{array}$ & $\begin{array}{l}0.698 \text { [range: } 0.524-0.873 \text {, dist: beta }(\alpha=4.8 \text {, } \\
\beta=2.1) \text { ] }\end{array}$ \\
\hline \multicolumn{3}{|l|}{ Utility inputs (time to death in days) $(11,12)$} \\
\hline$\geq 360$ & $\begin{array}{l}0.834 \text { [range: } 0.823-0.846, \text { dist: beta } \\
(\alpha=3,354, \beta=667.6)]\end{array}$ & $\begin{array}{l}0.842 \text { [range: } 0.823-0.861 \text {, dist: beta } \\
(\alpha=1,192, \beta=223.7)]\end{array}$ \\
\hline$[180,360)$ & $\begin{array}{l}0.765 \text { [range: } 0.743-0.786 \text {, dist: beta } \\
(\alpha=1,142.9, \beta=351.1)]\end{array}$ & $\begin{array}{l}0.814 \text { [range: } 0.795-0.833, \text { dist: beta } \\
(\alpha=1,311.5, \beta=299.7)]\end{array}$ \\
\hline$[30,180)$ & $\begin{array}{l}0.709 \text { [range: } 0.69-0.728, \text { dist: beta } \\
(\alpha=1,556.6, \beta=638.9)]\end{array}$ & $\begin{array}{l}0.737 \text { [range: } 0.717-0.756, \text { dist: beta } \\
(\alpha=1,443.2, \beta=515)]\end{array}$ \\
\hline $\begin{array}{l}\text { Maintenance chemotherapy with } \\
\text { pemetrexed per cycle }\end{array}$ & $\begin{array}{l}5,887 \text { [range: } 4,415-7,359, \text { dist: gamma } \\
(\alpha=45,994, \lambda=0.128)]\end{array}$ & - \\
\hline $\begin{array}{l}\text { Post-discontinuation treatment in } \\
\text { pembrolizumab treatment }\end{array}$ & $\begin{array}{l}\text { 13,097 [range: } 9,823-16,371 \text {, dist: gamma } \\
(\alpha=52,388, \lambda=0.25)]\end{array}$ & $\begin{array}{l}1,195 \text { [range: } 896-1,494, \text { dist: gamma } \\
(\alpha=4,780, \lambda=0.25)]\end{array}$ \\
\hline $\begin{array}{l}\text { Post-discontinuation treatment in } \\
\text { chemotherapy treatment }\end{array}$ & $\begin{array}{l}\text { 41,161 [range: } 30,871-51,451 \text {, dist: gamma } \\
(\alpha=164,644, \lambda=0.25)]\end{array}$ & $\begin{array}{l}15,763 \text { [range: } 11,822-19,704 \text {, dist: gamma } \\
(\alpha=63,052, \lambda=0.25)]\end{array}$ \\
\hline $\begin{array}{l}\text { Disease management in PFD state per } \\
21 \text {-days in 1st year }\end{array}$ & $\begin{array}{l}3,773 \text { [range: } 2,829-4,716 \text {, dist: gamma } \\
(\alpha=15,090, \lambda=0.25)]\end{array}$ & $\begin{array}{l}\text { 3,938 [range: } 2,953-4,922 \text {, dist: gamma } \\
(\alpha=15,752, \lambda=0.25)]\end{array}$ \\
\hline $\begin{array}{l}\text { Disease management in PFD state per } \\
21 \text {-days in } 2 \text { nd year }\end{array}$ & $\begin{array}{l}1,736 \text { [range: } 1,302-2,170, \text { dist: gamma } \\
(\alpha=6,945, \lambda=0.25)]\end{array}$ & $\begin{array}{l}2,088 \text { [range: } 1,566-2,611, \text { dist: gamma } \\
(\alpha=8,354, \lambda=0.25)]\end{array}$ \\
\hline $\begin{array}{l}\text { Disease management in PFD state per } \\
21 \text {-days in 3rd year }\end{array}$ & $\begin{array}{l}1,464 \text { [range: } 1,098-1,830, \text { dist: gamma } \\
(\alpha=5,855, \lambda=0.25)]\end{array}$ & $\begin{array}{l}922 \text { [range: } 691-1,152 \text {, dist: gamma } \\
(\alpha=3,687, \lambda=0.25)]\end{array}$ \\
\hline
\end{tabular}

Table 1 (continued) 
Table 1 (continued)

\begin{tabular}{|c|c|c|}
\hline Parameters & Nonsquamous non-small-cell lung cancer & Squamous non-small-cell lung cancer \\
\hline $\begin{array}{l}\text { Disease management in PD state per } \\
21 \text {-days in 1st year }\end{array}$ & $\begin{array}{l}3,785 \text { [range: } 2,839-4,731 \text {, dist: gamma } \\
(\alpha=15,139, \lambda=0.25)]\end{array}$ & $\begin{array}{l}4,345 \text { [range: } 3,259-5,432, \text { dist: gamma } \\
(\alpha=17,381, \lambda=0.25)]\end{array}$ \\
\hline $\begin{array}{l}\text { Disease management in PD state per } \\
21 \text {-days in 2nd year }\end{array}$ & $\begin{array}{l}2,967 \text { [range: } 2,225-3,709, \text { dist: gamma } \\
(\alpha=11,869, \lambda=0.25)]\end{array}$ & $\begin{array}{l}3,044 \text { [range: } 2,283-3,805, \text { dist: gamma } \\
(\alpha=12,175, \lambda=0.25)]\end{array}$ \\
\hline $\begin{array}{l}\text { Disease management in PD state per } \\
21 \text {-days in 3rd year }\end{array}$ & $\begin{array}{l}2,621 \text { [range: } 1,966-3,277 \text {, dist: gamma } \\
(\alpha=10,485, \lambda=0.25)]\end{array}$ & $\begin{array}{l}2,575 \text { [range: } 1,931-3,219, \text { dist: gamma } \\
(\alpha=10,301, \lambda=0.25)]\end{array}$ \\
\hline $\begin{array}{l}\text { Disease management in PD state per } \\
21 \text {-days in 4th to 5th year }\end{array}$ & $\begin{array}{l}2,462 \text { [range: } 1,846-3,077, \text { dist: gamma } \\
(\alpha=9,848, \lambda=0.25)]\end{array}$ & $\begin{array}{l}2,453 \text { [range: } 1,840-3,066, \text { dist: gamma } \\
(\alpha=9,811, \lambda=0.25)]\end{array}$ \\
\hline $\begin{array}{l}\text { Managing } A D R \text { (grade } \geq 3 \text { ) per patient related } \\
\text { to } I C I \text { treatment }\end{array}$ & $\begin{array}{l}2,020 \text { [range: } 1,515-2,525, \text { dist: gamma } \\
(\alpha=8,080, \lambda=0.25)]\end{array}$ & $\begin{array}{l}1,499 \text { [range: } 1,124-1,874 \text {, dist: gamma } \\
(\alpha=5,996, \lambda=0.25)]\end{array}$ \\
\hline $\begin{array}{l}\text { Managing ADR (grade } \geq 3 \text { ) per patient related } \\
\text { to chemotherapy }\end{array}$ & $\begin{array}{l}1,573 \text { [range: } 1,180-1,966, \text { dist: gamma } \\
(\alpha=6,292, \lambda=0.25)]\end{array}$ & $\begin{array}{l}1,259 \text { [range: } 944-1,574, \text { dist: gamma } \\
(\alpha=5,036, \lambda=0.25)]\end{array}$ \\
\hline Terminal care (last 30 days of life) & $\begin{array}{l}\text { 14,633 [range: } 10,975-18,291, \text { dist: gamma } \\
(\alpha=58,532, \lambda=0.25)]\end{array}$ & $\begin{array}{l}\text { 15,498 [range: } 11,624-19,373 \text {, dist: gamma } \\
(\alpha=61,992, \lambda=0.25)]\end{array}$ \\
\hline PD-L1 testing & $\begin{array}{l}111 \text { [range: } 83-138, \text { dist: gamma }(\alpha=437 \text {, } \\
\lambda=0.253) \text { ] }\end{array}$ & $\begin{array}{l}111 \text { [range: } 83-138, \text { dist: gamma }(\alpha=437, \\
\lambda=0.253)]\end{array}$ \\
\hline
\end{tabular}

*, the clinical inputs were based on the whole patients with unconfirmed PD-L1 TPS. AE, adverse event; HR, hazard ratio; PFS, progression-free survival; PFD, progression-free disease; PD, progressed disease; OS, overall survival.

As previous studies have done $(11,12)$, a time-to-death approach, reflecting the decline in cancer patients' qualityof-life, is used for modeling utilities. The utility scores of the $\geq 360,180$ to $<360,30$ to $<180$ and $<30$ day time-todeath in metastatic nonsquamous and squamous NSCLC were derived from published reports, which collected utility data by using EuroQOL-5D-3-level instrument in patients of the KEYNOTE-189 and KEYNOTE-407 trials (Table 1).

\section{Analysis}

In the base-case analysis, incremental cost-effectiveness ratio (ICER) was calculated as incremental cost per additional quality-adjusted life-year (QALY) gained between pembrolizumab plus chemotherapy and chemotherapy. Cost and QALYs were discounted at an annual rate of 3\% in the US and $5 \%$ in the Chinese context, respectively (22). We also estimated the incremental net-health benefit (INHB) based on the following formula: $\operatorname{INHB}(\lambda)=\left(\mu_{\mathrm{E} 1}-\mu_{\mathrm{E} 0}\right)-$ $\left(\mu_{\mathrm{C} 1}-\mu_{\mathrm{C} 0}\right) / \lambda=\Delta \mathrm{E}-\Delta \mathrm{C} / \lambda$, where $\mu_{\mathrm{Ci}}$ and $\mu_{\mathrm{Ei}}$ were the cost and effectiveness of pembrolizumab plus chemotherapy and chemotherapy (i=1) or chemotherapy and chemotherapy $(i=0)$, respectively, and $\lambda$ was the willingness-to-pay threshold of $\$ 150,000 / \mathrm{QALY}$ in the US $(23,24)$ and \$29,196/QALY (three times of per capita gross domestic product of China in 2018) in China. Subgroup analyses were performed in the prespecified subgroup as reported in the KEYNOTE-189 and KEYNOTE-407 trials by varying the HRs of OS between universal pembrolizumab and chemotherapy strategy in the whole patients with unconfirmed PD-L1 TPS, respectively $(7,8)$. The Markov model and statistical analyses were implemented in $\mathrm{R}$ software (http://www.r-project.org). The data used in this analysis is anonymous and therefore no informed consent was needed.

To evaluate the robustness of the base-case result, one- 
way and probabilistic sensitivity analyses (PSA) were conducted. One-way sensitivity analyses were conducted for all parameters, and the estimated range of each parameter was either based on the reported or estimated $95 \%$ confidence intervals in the referenced studies or determined by assuming a $25 \%$ change from the base-case value (Table 1). In the PSA, a Monte Carlo simulation of 1,000 iterations was generated by simultaneously sampling the key model parameters from the pre-specified distributions. Gamma distribution was selected for the cost parameters, log-normal distribution for hazard ratios, and beta distribution for probability, proportion and preference value parameters. Based on the data from 1,000 iterations, a cost-effectiveness acceptability curve (CEAC) was created to represent the likelihood that pembrolizumab plus chemotherapy would be considered cost-effective at various willingness-to-pay levels for health gains (QALYs).

\section{Results}

\section{Base-case analysis and subgroup analyses}

For metastatic nonsquamous NSCLC in the US context, universal pembrolizumab, TPS50 pembrolizumab and TPS1 pembrolizumab strategy provided an additional $1.168,0.388$ and 0.777 QALYs with an incremental cost of $\$ 142,773, \$ 55,594$ and $\$ 99,219$ comparing with chemotherapy in the whole patients with unconfirmed PD-L1 TPS, which resulted in the ICER of $\$ 122,248$, $\$ 143,282$ and $\$ 127,661 /$ QALY, respectively. The INHBs of universal pembrolizumab, TPS50 pembrolizumab and TPS1 pembrolizumab strategy over chemotherapy were 0.216, 0.017 and 0.116 QALYs at the threshold of $\$ 150,000 /$ QALY, respectively (Table 2). The subgroup analysis by varying the HRs of OS found that universal pembrolizumab showed the trend of gaining health benefits in most of the subgroups except the male patients (Figure 2). The INHBs in the subgroups with the respect to the health benefit varied from -0.07 (range, -0.47 to 0.48 , probabilities of cost-effectiveness: $25.7 \%$ ) in male patients to 2.21 (range, 0.34 to 4.05 , probabilities of cost-effectiveness: $100 \%$ ) in never-smoking patients. In three subgroups with confirmed PD-L 1 TPS $<1 \%, 1 \%$ to $49 \%$ and $\geq 50 \%$, the ICERs of pembrolizumab over chemotherapy strategy were $\$ 111,763$, \$112,088 and \$142,997/QALY, respectively.

For metastatic squamous NSCLC in the US context, universal pembrolizumab, TPS50 pembrolizumab and TPS1 pembrolizumab strategy provided an additional
$0.988,0.272$ and 0.667 QALYs with an incremental cost of $\$ 84,934, \$ 1,175$ and $\$ 46,045$ comparing with chemotherapy in the whole patients with unconfirmed PD-L1 TPS, which resulted in the ICER of $\$ 121,375$, $\$ 131,495$ and $\$ 121,554 /$ QALY, respectively. The INHBs of universal pembrolizumab, TPS50 pembrolizumab and TPS1 pembrolizumab strategy over chemotherapy were 0.195, 0.038 and 0.133 QALYs at the threshold of $\$ 150,000 /$ QALY, respectively (Table 2). The subgroup analysis by varying the HRs of OS found that universal pembrolizumab showed the trend of gaining health benefits in most of the subgroups except the male patients (Figure 2). The INHBs in the subgroups with the respect to the health benefit varied from -0.12 (range, -0.47 to 0.50 , probabilities of cost-effectiveness: $26.8 \%$ ) in those age $\geq 65$ years old to 0.88 (range, -0.23 to 2.4 , probabilities of cost-effectiveness: $92.1 \%$ ) in female patients. In three subgroups with confirmed PD-L1 TPS $<1 \%, 1 \%$ to $49 \%$ and $\geq 50 \%$, the ICERs of pembrolizumab over chemotherapy strategy were $\$ 121,326, \$ 113,780$ and $\$ 131,136 /$ QALY, respectively.

For metastatic nonsquamous and squamous NSCLC in the Chinese context, the ICER of universal pembrolizumab, TPS50 pembrolizumab and TPS1 pembrolizumab strategies were all higher than $\$ 40,000 / \mathrm{QALY}$ in comparison with chemotherapy (Table S4).

\section{Sensitivity analyses}

The one-way sensitivity analyses revealed that the HR of OS between universal pembrolizumab and chemotherapy strategy played a vital role in model outcomes regardless of histology in the US context (Figure 3). When its upper boundaries were adopted, the ICERs would exceed the threshold of \$150,000/QALY. Other parameters, such as the cost of post-discontinuation treatment and related disease management and utility values, had a medium or small impact on the outcome. In general, the model results were robust to the adjustment of parameters.

For metastatic nonsquamous NSCLC in the US context, the CEAC showed nearly $86 \%, 54 \%$ and $75 \%$ probabilities of universal pembrolizumab, TPS50 pembrolizumab and TPS1 pembrolizumab strategy being a cost-effective strategy compared with chemotherapy at the threshold of $\$ 150,000 /$ QALY in the whole patients with unconfirmed PD-L 1 TPS (Figure $4 A$ ), and $88 \%, 82 \%$ and $55 \%$ probabilities of pembrolizumab being a cost-effective strategy compared with chemotherapy in three subgroups 
Table 2 Summary of cost (\$) and outcome results in base-case analysis

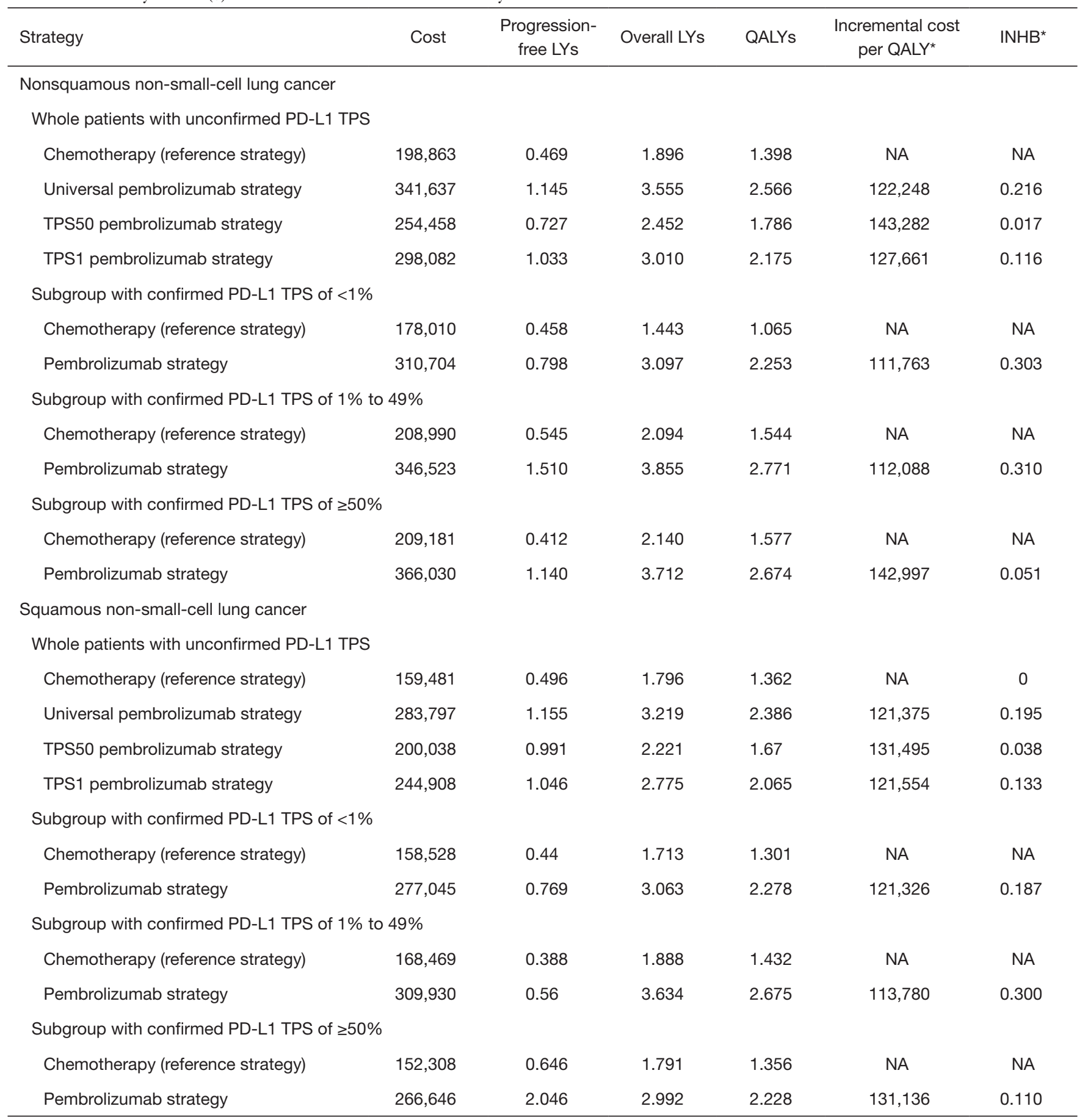

*, comparing with reference strategy. QALY, quality-adjusted-life year; PD-L1, programmed cell death 1 ligand 1; TPS, tumor proportion score.

with confirmed PD-L1 TPS $<1 \%, 1 \%$ to $49 \%$ and $\geq 50 \%$ (Figure 4B).

For metastatic squamous NSCLC in the US context, the CEAC showed nearly $83 \%, 60 \%$ and $77 \%$ probabilities of universal pembrolizumab, TPS50 pembrolizumab and TPS1 pembrolizumab strategy being a cost-effective 


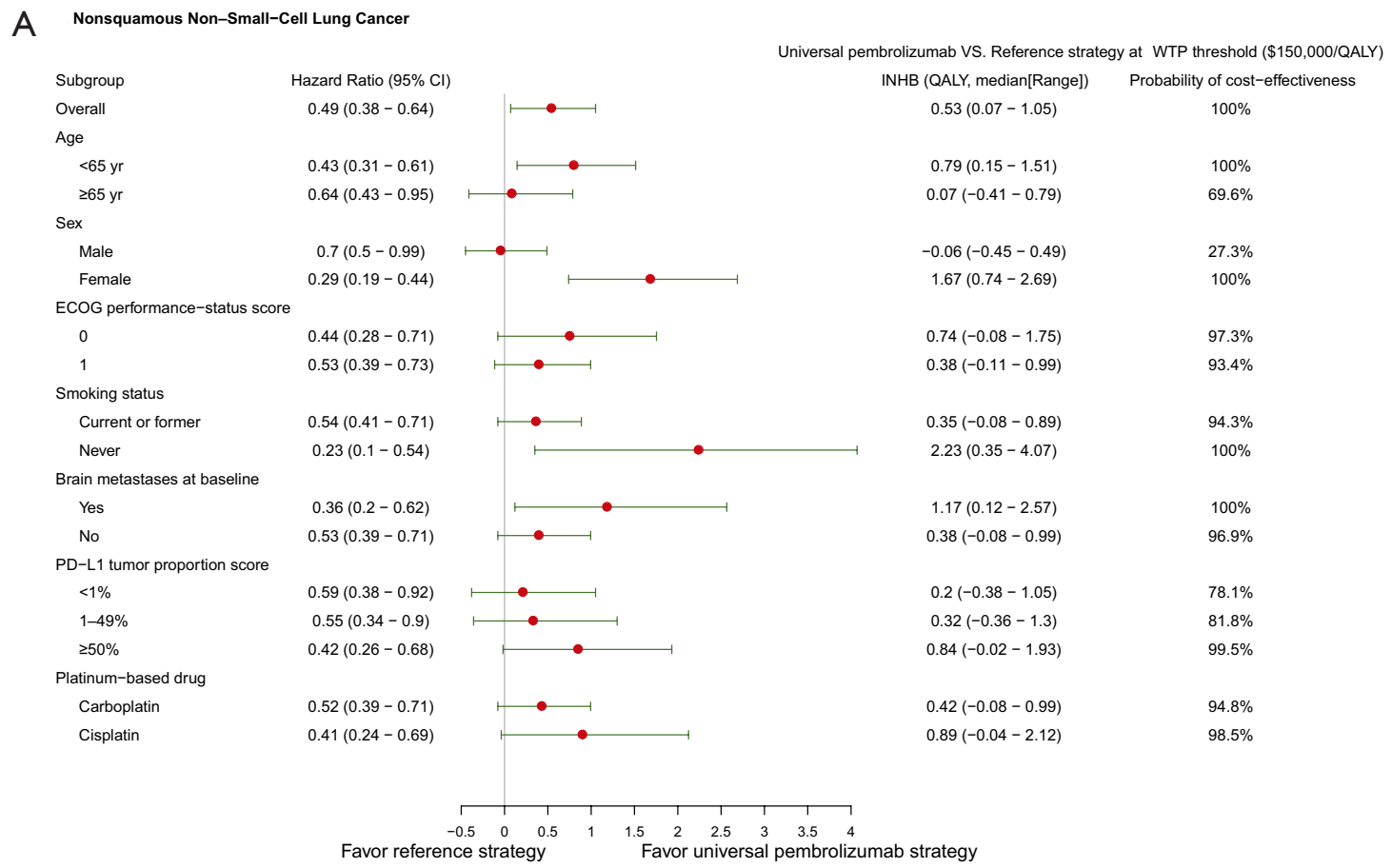

B Squamous Non-Small-Cell Lung Cancer

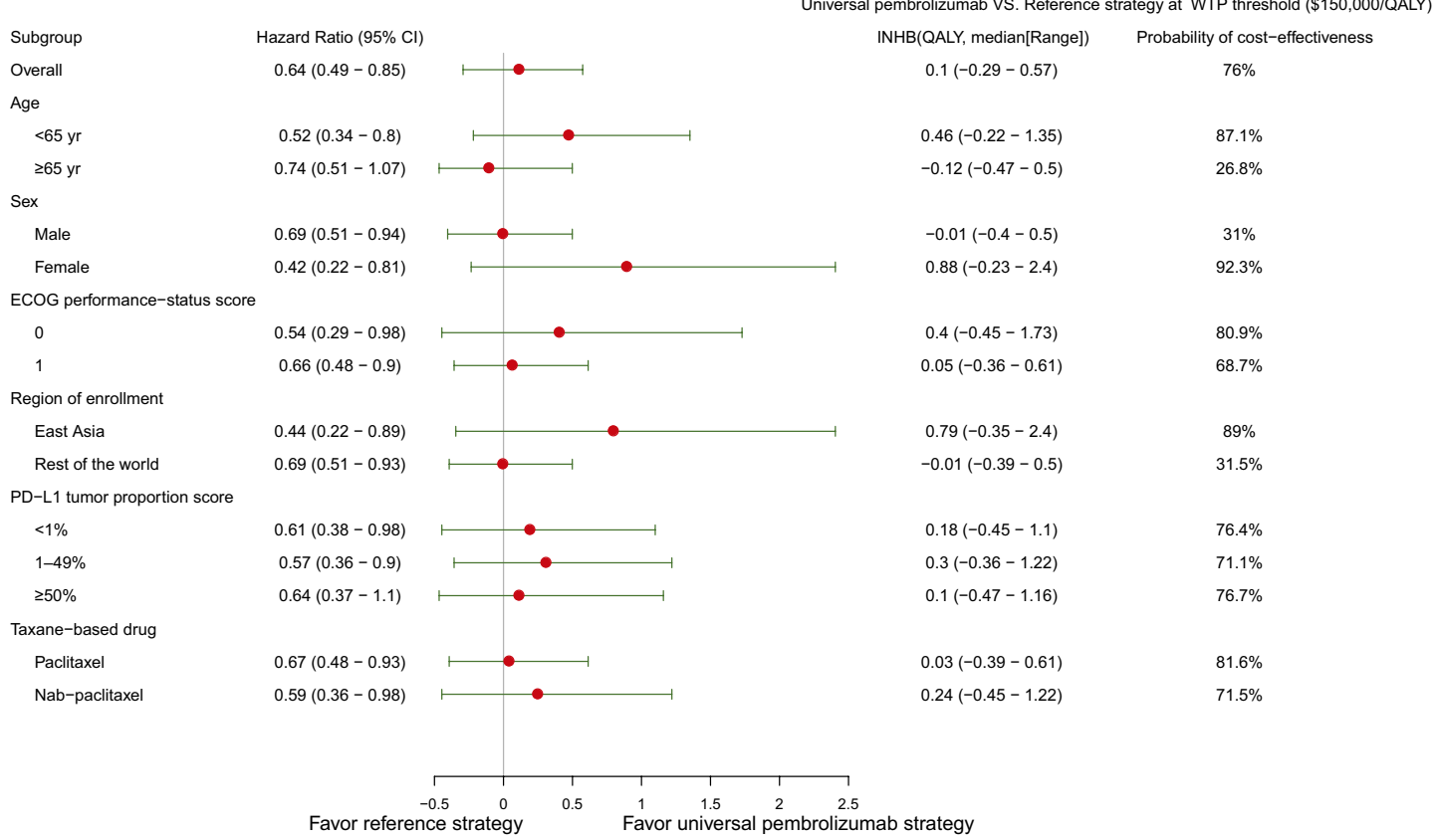

Figure 2 Subgroup analysis of incremental net health benefits (INHB) and probabilities of cost-effectiveness of universal pembrolizumab strategy versus reference strategy by varying the hazard ratios (HRs) of OS in whole patients with unconfirmed PD-L1 TPS metastatic nonsquamous NSCLC (A) and squamous NSCLC (B). The vertical line indicates the point of no effect (INHB =0), the red circle indicates the median INHB, and the green bar indicates the ranges of INHB adjusted by the HRs. OS, overall survival; PD-L1, programmed cell death 1 ligand 1; TPS, tumor proportion score; NSCLC, non-small-cell lung cancer. 
A Nonsquamous Non-Small-Cell Lung Cancer

HR of OS of universal pembrolizumab versus standard chemotherapy Cost of pembrolizumab per $200 \mathrm{mg}$ Duration of chemotherapy Costs of post-discontinuation treatment in standard chemotherapy strategy Year of time horizon

Cost of PFD related to disease management per 21-days in 1st year HR of PFS of universal pembrolizumab versus standard chemotherapy Costs of post-discontinuation treatment in universal pembrolizumab strategy Age of patient Utility of time-to-death $\geq 360$ days Cost of PFD related to disease management per 21-days in 2nd year Cost of PD related to disease management per 21-days in $>5$ th year Cost of PD related to disease management per 21-days in 2nd year Cost of PD related to disease management per 21-days in 1st year Cost of PD related to disease management per 21-days in 4th-5th year

Cost of maintenance chemotherapy with pemetrexed per 21-days Cost of PD related to disease management per 21-days in 3rd year Cost of PFD related to disease management per 21-days in 3rd year Cost of PFD related to disease management per 21-days in 4th-5th year Probability of ADR (grade $\geq 3$ ) related to pembrolizumab treatment Cost of managing ADR (grade $\geq 3$ ) per patient related to pembrolizumab treatment Cost of PFD related to disease management per 21 -days in $>5$ th year Utility of time-to-death 180-360 days Cost of terminal Care (last 30 days of life) Utility of time-to-death $30-180$ days

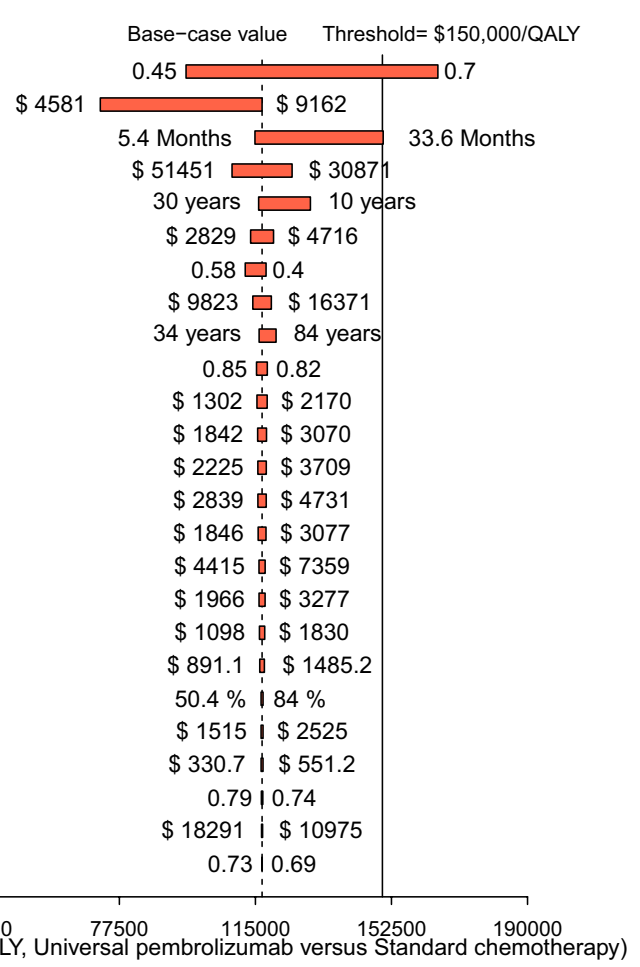

B Squamous Non-Small-Cell Lung Cancer

HR of OS of universal pembrolizumab versus standard chemotherapy Cost of pembrolizumab per $200 \mathrm{mg}$ Year of time horizon

Costs of post-discontinuation treatment in standard chemotherapy strategy Cost of PFD related to disease management per 21-days in 1st year Utility of time-to-death $\geq 360$ days HR of PFS of universal pembrolizumab versus standard chemotherapy Age of patient

Cost of PFD related to disease management per 21-days in 2nd year Cost of PD related to disease management per 21-days in 1st year Cost of PD related to disease management per 21-days in 2nd year Cost of PD related to disease management per 21-days in 3rd year Cost of PD related to disease management per 21-days in 4th-5th year Cost of PFD related to disease management per 21-days in 4th-5th year

Cost of PFD related to disease management per 21-days in 3rd year Costs of post-discontinuation treatment in universal pembrolizumab strategy Utility of time-to-death $180-360$ days

Cost of PD related to disease management per 21-days in $>5$ th year Cost of terminal Care (last 30 days of life) Utility of time-to-death 30-180 days

Probability of ADR (grade $\geq 3$ ) related to pembrolizumab treatment Cost of managing ADR (grade $\geq 3$ ) per patient related to pembrolizumab treatment

Base-case value Threshold $=\$ 150,000 /$ QALY

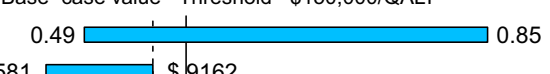

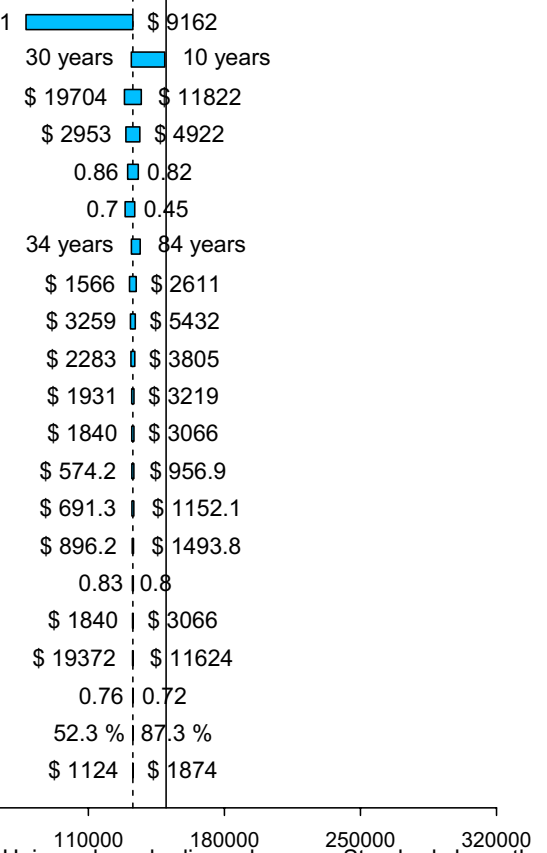

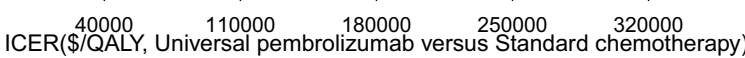

Figure 3 Tornado diagram of one-way sensitivity analyses of universal pembrolizumab versus chemotherapy (reference strategy) in the whole patients with unconfirmed PD-L1 TPS metastatic nonsquamous NSCLC (A) and squamous NSCLC (B). PD-L1, programmed cell death 1 ligand 1; TPS, tumor proportion score; NSCLC, non-small-cell lung cancer. 

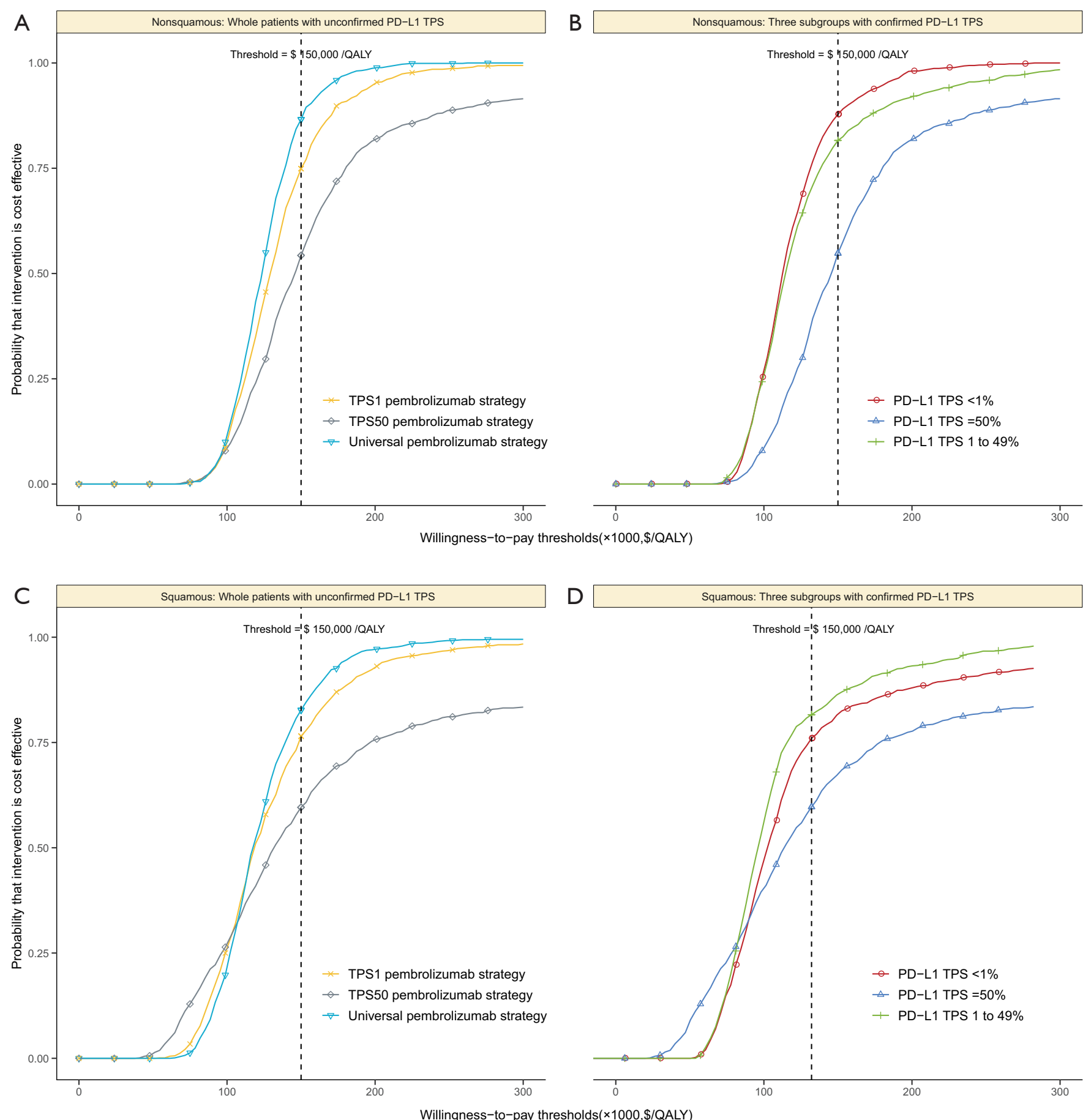

Figure 4 Cost-effectiveness acceptability curves of universal pembrolizumab, TPS50 pembrolizumab and TPS1 pembrolizumab strategy versus chemotherapy (reference strategy) for metastatic nonsquamous and squamous NSCLC in the whole patients with unconfirmed PDL1 TPS (A and C), and pembrolizumab strategy versus chemotherapy (reference strategy) in three subgroups with confirmed PD-L1 TPS $<1 \%, 1 \%$ to $49 \%$ and $\geq 50 \%$ (B and D). PD-L1, programmed cell death 1 ligand 1; TPS, tumor proportion score; NSCLC, non-small-cell lung cancer. 
strategy compared with chemotherapy at the threshold of $\$ 150,000 /$ QALY in the whole patients with unconfirmed PD-L1 TPS (Figure $4 C$ ), and $76 \%, 82 \%$ and $60 \%$ probabilities of pembrolizumab being a cost-effective strategy compared with chemotherapy in three subgroups with confirmed PD-L1 TPS $<1 \%, 1 \%$ to $49 \%$ and $\geq 50 \%$ (Figure 4D).

For metastatic nonsquamous and squamous NSCLC in the Chinese context, the CEAC showed lower than $10 \%$ probabilities of universal pembrolizumab, TPS50 pembrolizumab and TPS1 pembrolizumab strategy being a cost-effective strategy compared with chemotherapy at the threshold of $\$ 29,196 /$ QALY in the whole patients with unconfirmed PD-L1 TPS and three subgroups with confirmed PD-L1 TPS $<1 \%, 1 \%$ to $49 \%$ and $\geq 50 \%$.

\section{Discussion}

To our knowledge, this is the first analysis to address the unmet need for an economic assessment of PD-L1 categories-directed pembrolizumab treatment in the first-line setting. When treatment was guided according to the PD-L1 TPS after PD-L1 expression status was tested, adding pembrolizumab for only those with PD-L1 TPS $\geq 1 \%$ and $\geq 50 \%$ did not show the improvement of cost-effectiveness in comparison with routinely adding pembrolizumab treatment for all patients. Although the ICERs of universal pembrolizumab, TPS50 pembrolizumab and TPS1 pembrolizumab strategy versus chemotherapy were comparable, the universal pembrolizumab strategy gained the greatest incremental QALYs and INHB. The sensitivity analysis confirmed that these results are generally robust. These findings suggest that routinely adding pembrolizumab to chemotherapy without PD-L1 testing is a favorable option for previously untreated metastatic nonsquamous and squamous NSCLC without EGFR or ALK mutations. This finding could be supported by the results in the subgroups prespecified by the KEYNOTE-189 and KEYNOTE-407 trials $(7,8)$, which showed the addition of pembrolizumab treatment are costeffective in these subgroups. However, in the developing setting, adding pembrolizumab to chemotherapy is not a cost-effective option because its ICERs of pembrolizumab plus chemotherapy against chemotherapy were higher than \$100,000/QALY regardless of the histology and TPS subgroups, which highly exceed the willingness-to-pay threshold of \$29,196/QALY in the Chinese context.

Our finding was consistent with two recent economic analyses, which found that pembrolizumab plus chemotherapy generated an ICER of \$104,823/QALY and \$86,293/QALY comparing with chemotherapy strategy in the overall non-squamous and squamous NSCLC population from a US healthcare payer perspective, respectively $(11,12)$. However, adding pembrolizumab to chemotherapy for non-squamous NSCLC with PD-L1 $<1 \%$ is exceeding $\$ 150,000$ per QALY, which is distinguished with our results. A possible explanation for this variation could be that the incremental health outcomes in Insinga's evaluation were lower than ours due to the different method of tracking the survival probabilities among three subgroups, where the same estimation in Insinga's evaluation was applied for three subgroups and the full trial population (12). As shown by the results of KEYNOTE-189 trial, health outcomes in PD-L1 $\geq 50 \%$ subgroup receiving chemotherapy is superior to the PD-L1 $<1 \%$ subgroup. However, Insinga's evaluation showed the PD-L $1<1 \%$ subgroup had the longer life-years and QALYs than the PD-L1 $\geq 50 \%$ subgroup (12), which in turn had an underestimation effect of pembrolizumab plus chemotherapy on the incremental health outcomes in PD-L $1<1 \%$ subgroup. By using the estimated HRs of PFS and OS between three subgroups and the whole patients, our analysis captured the distinguishable health outcomes among three subgroups.

One recent economic evaluation found that the use of PD-L1 expression as a biomarker increases the costeffectiveness of immunotherapy for second-line treatment of metastatic/metastatic NSCLC from a US healthcare payer perspective (10), which is coherent with our finding in the squamous NSCLC. However, it is discordant with the nonsquamous NSCLC that adding pembrolizumab for all patients without PD-L1 testing was more cost-effective than the PDL1-directed pembrolizumab treatment, which might be explained by the fact that the PD-L $1<1 \%$ subgroup in nonsquamous NSCLC had the more favorable economic outcomes than those with PD-L1 TPS $\geq 50 \%$. The potential reason is that the lower incremental cost and higher incremental health outcomes of pembrolizumab versus chemotherapy strategy in PD-L1 $<1 \%$ subgroup than those with PD-L1 TPS $\geq 50 \%$, which was yielded by the relatively higher HR of PFS and lower OS of pembrolizumab versus chemotherapy strategy in PD-L1 $<1 \%$ subgroup (6). The higher HR of PFS led to the shorter duration and lower cost of pembrolizumab treatment.

The nature of pembrolizumab plus chemotherapy to prolong the OS was a major driver of economic outcomes. 
The results of one-way sensitivity analysis revealed that the HR of OS is a substantial model input, which suggested that the addition of pembrolizumab would become more cost-effective in patients with more favorable $\mathrm{HR}$ of OS, such as the female patients and age $<65$ years old. However, in some patients with more unfavorable HR of OS who have a high risk of death, such as those aged $\geq 65$ years, the addition of pembrolizumab might be less cost-effective. The cost of pembrolizumab was also found to be a considerable influential factor. When the cost of pembrolizumab decreased by $50 \%$, the ICER for the addition of pembrolizumab decreased to be close to $\$ 50,000 /$ QALY. Recently, the US government has proposed indexing the prices that Medicare pays for drugs to those paid by health systems in other developed countries, to help bring down the relatively high prices paid by US patients (25). Once it is enacted or implemented, the initiative might lead to a reduction in the price of pembrolizumab and achieving a more favorable economic outcome.

There are several weaknesses with the analysis. Firstly, due to the lack of head-to-head data, the discrepancy of trial design and different techniques of biomarker testing, we did not include other ICIs regimens, such as nivolumab plus ipilimumab and atezolizumab plus chemotherapy, which have also shown favorable health benefits in the first-line setting for metastatic NSCLC $(26,27)$. Secondly, health benefits beyond the observation time of the KEYNOTE-189 and KEYNOTE-407 trials were assumed through the fitting of parametric distributions to the reported PFS and OS data, which might result in uncertainty in the model outputs although the predicted and observed data were validated. Thirdly, we did not measure the budget impact of adding pembrolizumab on society, which usually performed in addition to a cost-effectiveness analysis for estimating the financial consequences of adopting a new intervention (28). For example, because about annually 64,901 new NSCLC patients would be eligible for 17.9 first-line treatment cycles of pembrolizumab (29), wide first-line prescription of pembrolizumab might intensively raise the financial burden. However, because the findings of this evaluation reflected the general clinical conditions of managing metastatic NSCLC, it might be a valuable reference for physicians and policy-makers.

These estimates demonstrated that adding pembrolizumab to chemotherapy as a first-line strategy was the cost-effective option in the US, and could be directly administered for treating metastatic nonsquamous and squamous NSCLC harboring no EGFR or ALK mutations and without PDL1 expression information. However, pembrolizumab plus chemotherapy is not a cost-effective option in the Chinese context. Reducing the price of pembrolizumab could improve the economic outcomes. These findings might contribute to aiding clinicians in making the optimal decision for the treatment of metastatic NSCLC.

\section{Acknowledgments}

Funding: None.

\section{Footnote}

Reporting Checklist: The authors have completed the CHEERS Reporting Checklist. Available at http://dx.doi. org/10.21037/tlcr-19-605

Data Sharing Statement: Available at http://dx.doi. org/10.21037/tlcr-19-605

Conflicts of Interest: All authors have completed the ICMJE uniform disclosure form (available at http://dx.doi. org/10.21037/tlcr-19-605). The authors have no conflicts of interest to declare.

Ethical Statement: The authors are accountable for all aspects of the work in ensuring that questions related to the accuracy or integrity of any part of the work are appropriately investigated and resolved. This study was based on a literature review and modeling techniques; this study did not require approval by an institutional research ethics board.

Open Access Statement: This is an Open Access article distributed in accordance with the Creative Commons Attribution-NonCommercial-NoDerivs 4.0 International License (CC BY-NC-ND 4.0), which permits the noncommercial replication and distribution of the article with the strict proviso that no changes or edits are made and the original work is properly cited (including links to both the formal publication through the relevant DOI and the license). See: https://creativecommons.org/licenses/by-nc-nd/4.0/.

\section{References}

1. Anonymous. Global, regional, and national disabilityadjusted life-years (DALYs) for 359 diseases and injuries 
and healthy life expectancy (HALE) for 195 countries and territories, 1990-2017: a systematic analysis for the Global Burden of Disease Study 2017. Lancet 2018;392:1859-922.

2. Griesinger F, Korol EE, Kayaniyil S, et al. Efficacy and safety of first-line carboplatin-versus cisplatin-based chemotherapy for non-small cell lung cancer: A metaanalysis. Lung Cancer 2019;135:196-204.

3. Zhou Y, Chen C, Zhang X, et al. Immune-checkpoint inhibitor plus chemotherapy versus conventional chemotherapy for first-line treatment in advanced nonsmall cell lung carcinoma: a systematic review and metaanalysis. J Immunother Cancer 2018;6:155.

4. Gubens MA, Davies M. NCCN Guidelines Updates: New Immunotherapy Strategies for Improving Outcomes in Non-Small Cell Lung Cancer. J Natl Compr Canc Netw 2019;17:574-8.

5. Peters S, Reck M, Smit EF, et al. How to make the best use of immunotherapy as first-line treatment of advanced/ metastatic non-small-cell lung cancer. Ann Oncol 2019;30:884-96.

6. Gadgeel SM, Garassino MC, Esteban E, et al. KEYNOTE-189: Updated OS and progression after the next line of therapy (PFS2) with pembrolizumab (pembro) plus chemo with pemetrexed and platinum vs placebo plus chemo for metastatic nonsquamous NSCLC. J Clin Oncol 2019;37:9013.

7. Gandhi L, Rodríguez-Abreu D, Gadgeel S, et al. Pembrolizumab plus Chemotherapy in Metastatic Non-Small-Cell Lung Cancer. New Engl J Med 2018;378:2078-92.

8. Paz-Ares L, Luft A, Vicente D, et al. Pembrolizumab plus Chemotherapy for Squamous Non-Small-Cell Lung Cancer. N Engl J Med 2018;379:2040-51.

9. Vecchiarelli S, Passiglia F, D'Incecco A, et al. Circulating programmed death ligand-1 (cPD-L1) in non-small-cell lung cancer (NSCLC). Oncotarget 2018;9:17554-63.

10. Aguiar PN, Perry LA, Penny-Dimri J, et al. The effect of PD-L1 testing on the cost-effectiveness and economic impact of immune checkpoint inhibitors for the secondline treatment of NSCLC. Ann Oncol 2017;28:2256-63.

11. Insinga RP, Vanness DJ, Feliciano JL, et al. Costeffectiveness of pembrolizumab in combination with chemotherapy versus chemotherapy and pembrolizumab monotherapy in the first-line treatment of squamous nonsmall-cell lung cancer in the US. Curr Med Res Opin 2019;35:1241-56.

12. Insinga RP, Vanness DJ, Feliciano JL, et al. Costeffectiveness of pembrolizumab in combination with chemotherapy in the 1st line treatment of non-squamous NSCLC in the US. J Med Econ 2018;21:1191-205.

13. Guyot P, Ades AE, Ouwens MJ, et al. Enhanced secondary analysis of survival data:reconstructing the data from published Kaplan-Meier survival curves. BMC Med Res Methodol 2012;12:9.

14. Ouwens MJNM, Mukhopadhyay P, Zhang Y, et al. Estimating Lifetime Benefits Associated with ImmunoOncology Therapies: Challenges and Approaches for Overall Survival Extrapolations. Pharmacoeconomics 2019;39:1129-38.

15. Treat J, Edelman MJ, Belani CP, et al. A retrospective analysis of outcomes across histological subgroups in a three-arm phase III trial of gemcitabine in combination with carboplatin or paclitaxel versus paclitaxel plus carboplatin for advanced non-small cell lung cancer. Lung Cancer 2010;70:340-6.

16. Mok TSK, Wu Y, Kudaba I, et al. Pembrolizumab versus chemotherapy for previously untreated, PD-L1-expressing, locally advanced or metastatic non-small-cell lung cancer (KEYNOTE-042): a randomised, open-label, controlled, phase 3 trial. Lancet 2019;393:1819-30.

17. Anonymous. US Department of Labor. Calculators. Available online: https://www.bls.gov/data/inflation calculator.htm. [Accessed January 5, 2019].

18. Anonymous. RED BOOK Online. Available online: http:// www.micromedexsolutions.com. [Accessed 24 March 2019].

19. Hornberger J, Hirsch FR, Li Q, et al. Outcome and economic implications of proteomic test-guided secondor third-line treatment for advanced non-small cell lung cancer: extended analysis of the PROSE trial. Lung Cancer 2015;88:223-30.

20. Yu TM, Morrison C, Gold EJ, et al. Budget Impact of Next-Generation Sequencing for Molecular Assessment of Advanced Non-Small Cell Lung Cancer. Value Health 2018;21:1278-85.

21. Criss SD, Weaver DT, Sheehan DF, et al. Effect of PDL1 testing on the cost-effectiveness and budget impact of pembrolizumab for advanced urothelial carcinoma of the bladder in the United States. Urol Oncol 2019;37:180. e11-8.

22. Sanders GD, Neumann PJ, Basu A, et al. Recommendations for Conduct, Methodological Practices, and Reporting of Cost-effectiveness Analyses: Second Panel on Cost-Effectiveness in Health and Medicine. JAMA 2016;316:1093-103.

23. Craig BA, Black MA. Incremental cost-effectiveness ratio and incremental net-health benefit:two sides of the 
same coin. Expert Rev Pharmacoecon Outcomes Res 2001;1:37-46.

24. Stinnett AA, Mullahy J. Net health benefits:a new framework for the analysis of uncertainty in costeffectiveness analysis. Med Decis Making 1998;18:S68-80.

25. Dyer O. US drug prices should be tied to foreign prices to tackle "global freeloading," says Trump. BMJ 2018;363:k4542.

26. Hellmann MD, Ciuleanu T, Pluzanski A, et al. Nivolumab plus Ipilimumab in Lung Cancer with a High Tumor Mutational Burden. New Engl J Med 2018;378:2093-104.

27. West H, Mccleod M, Hussein M, et al. Atezolizumab in combination with carboplatin plus nab-paclitaxel

Cite this article as: $\mathrm{Wu} \mathrm{B}, \mathrm{Lu} \mathrm{S}$. The effect of PD-L1 categories-directed pembrolizumab plus chemotherapy for newly diagnosed metastatic non-small-cell lung cancer: a costeffectiveness analysis. Transl Lung Cancer Res 2020;9(5):17701784. doi: $10.21037 /$ tlcr-19-605 chemotherapy compared with chemotherapy alone as firstline treatment for metastatic non-squamous non-smallcell lung cancer (IMpower130): a multicentre, randomised, open-label, phase 3 trial. Lancet Oncol 2019;20:924-37.

28. Sullivan SD, Mauskopf JA, Augustovski F, et al. Budget impact analysis-principles of good practice: report of the ISPOR 2012 Budget Impact Analysis Good Practice II Task Force. Value Health 2014;17:5-14.

29. Goldstein DA, Gordon N, Davidescu M, et al. A Phamacoeconomic Analysis of Personalized Dosing vs Fixed Dosing of Pembrolizumab in Firstline PD-L1Positive Non-Small Cell Lung Cancer. JNCI 2017;109. 
Table S1 Survival model parameters fitting to the PFS and OS data from KEYNOTE-189 and KEYNOTE-407 trials

\begin{tabular}{|c|c|c|c|c|c|c|}
\hline \multirow{2}{*}{ Trials and populations } & \multicolumn{3}{|c|}{ PFS } & \multicolumn{3}{|c|}{ Os } \\
\hline & Model & Parameter & AIC & Model & Parameter & AIC \\
\hline \multicolumn{7}{|l|}{ KEYNOTE-189 trial } \\
\hline \multicolumn{7}{|c|}{ Whole patients with unconfirmed PD-L1 TPS } \\
\hline Pembrolizumab plus chemotherapy & Gamma & Shape $=1.4661 ;$ rate $=0.0904$ & 1873.05 & Mixed cure model with Gompertz & Theta $=0.6019 ;$ shape $=0.0894 ;$ rate $=0.0346$ & 1241.31 \\
\hline Chemotherapy & Log-normal & Meanlog $=1.9509 ;$ sdlog $=0.9444$ & 1083.14 & Mixed cure model with Weibull & Theta $=0.4023 ;$ shape $=1.5979 ;$ scale $=11.1068$ & 897.70 \\
\hline \multicolumn{7}{|c|}{ Subgroup with confirmed PD-L1 TPS of $<1 \%$} \\
\hline Pembrolizumab plus chemotherapy & Log-normal & Meanlog $=2.1661 ;$ sdlog $=0.9333$ & 639.15 & Exp & Rate $=0.0264$ & 456.18 \\
\hline Chemotherapy & Log-normal & Meanlog =1.956; sdlog =0.8955 & 351.90 & Log-normal & Meanlog =2.749; sdlog =1.2256 & 291.22 \\
\hline \multicolumn{7}{|c|}{ Subgroup with confirmed PD-L1 TPS of $1 \%$ to $49 \%$} \\
\hline Pembrolizumab plus chemotherapy & Log-logistic & Shape $=1.6079 ;$ scale $=13.5793$ & 550.35 & Mixed cure model with Weibull & Theta $=0.6321 ;$ shape $=1.4845 ;$ scale $=13.4376$ & 363.45 \\
\hline Chemotherapy & Log-logistic & Shape $=2.1047 ;$ scale $=8.4391$ & 291.20 & Mixed cure model with Gompertz & Theta $=0.4666 ;$ shape $=0.1891 ;$ rate $=0.0208$ & 241.85 \\
\hline \multicolumn{7}{|c|}{ Subgroup with confirmed PD-L1 TPS of $\geq 50 \%$} \\
\hline Pembrolizumab plus chemotherapy & Log-normal & Meanlog $=2.7938 ;$ sdlog $=1.3274$ & 573.49 & Mixed cure model with Exp & Theta $=0.4777 ;$ rate $=0.0356$ & 343.80 \\
\hline Chemotherapy & Gompertz & Shape $=0.0029 ;$ rate $=0.1024$ & 369.26 & Mixed cure model with Weibull & Theta $=0.4403 ;$ shape $=1.6597 ;$ scale $=10.0783$ & 298.21 \\
\hline \multicolumn{7}{|l|}{ KEYNOTE-407 trial } \\
\hline \multicolumn{7}{|c|}{ Whole patients with unconfirmed PD-L1 TPS } \\
\hline Pembrolizumab plus chemotherapy & Royston/Parmar spline & 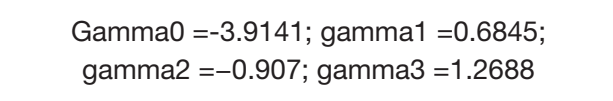 & 1149.42 & Royston/Parmar spline & 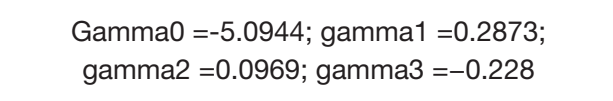 & 811.27 \\
\hline Chemotherapy & Log-logistic & Shape $=1.8725 ;$ scale $=7.0535$ & 1294.25 & Mixed cure model with Gompertz & Theta $=0.3285 ;$ shape $=0.0871 ;$ rate $=0.0363$ & 1014.34 \\
\hline \multicolumn{7}{|c|}{ Subgroup with confirmed PD-L1 TPS of $<1 \%$} \\
\hline Pembrolizumab plus chemotherapy & Weibull & Shape $=1.3007 ;$ scale $=14.1579$ & 403.01 & Gompertz & Shape $=0.0577 ;$ rate $=0.016$ & 279.48 \\
\hline Chemotherapy & Log-logistic & Shape $=2.1166 ;$ scale $=6.6265$ & 508.39 & Mixed cure model with Gompertz & Theta $=0.3034 ;$ shape $=0.1604 ;$ rate $=0.0196$ & 363.49 \\
\hline \multicolumn{7}{|c|}{ Subgroup with confirmed PD-L1 TPS of $1 \%$ to $49 \%$} \\
\hline Pembrolizumab plus chemotherapy & Log-logistic & Shape $=1.4189 ;$ scale $=11.4898$ & 425.05 & Mixed cure model with Gompertz & Theta $=0.0035 ;$ shape $=0.0634 ;$ rate $=0.0139$ & 302.59 \\
\hline Chemotherapy & Weibull & Shape $=1.6288 ;$ scale $=9.7936$ & 489.05 & Exp & Rate $=0.0373$ & 379.33 \\
\hline \multicolumn{7}{|c|}{ Subgroup with confirmed PD-L1 TPS of $\geq 50 \%$} \\
\hline Pembrolizumab plus chemotherapy & Weibull & Shape $=1.5796 ;$ scale $=14.3775$ & 299.79 & Mixed cure model with Exp & Theta $=0.0033 ;$ rate $=0.0263$ & 217.50 \\
\hline Chemotherapy & Weibull & Theta $=0.0781 ;$ shape $=1.5134 ;$ scale $=6.3663$ & 345.17 & Exp & Rate $=0.0383$ & 249.26 \\
\hline
\end{tabular}

PFS, progression-free survival; OS, overall survival; AIC, Akaike information criterion; PD-L1, programmed cell death 1 ligand 1; TPS, tumor proportion score. 
A

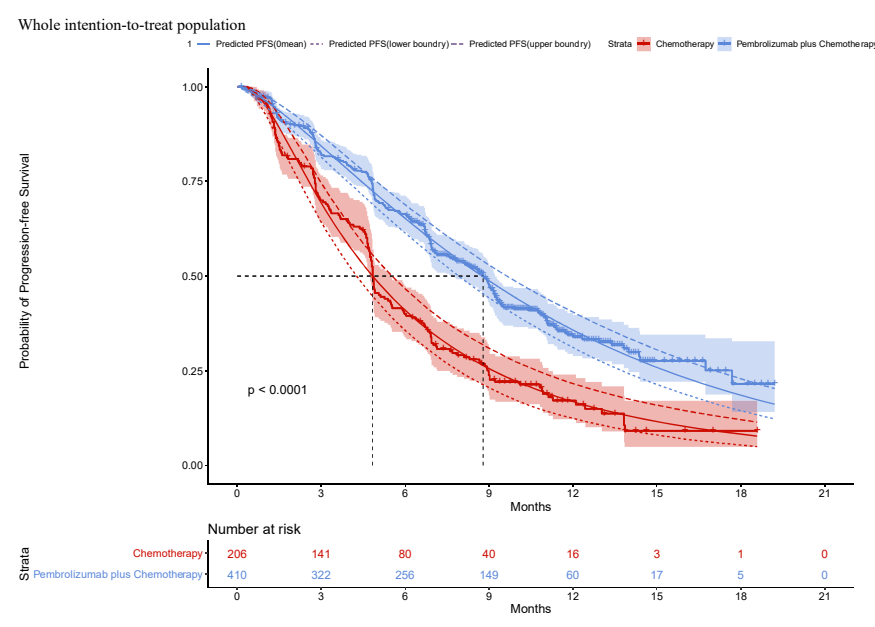

\section{C}

Tumor Proportion Score of 1 to $49 \%$

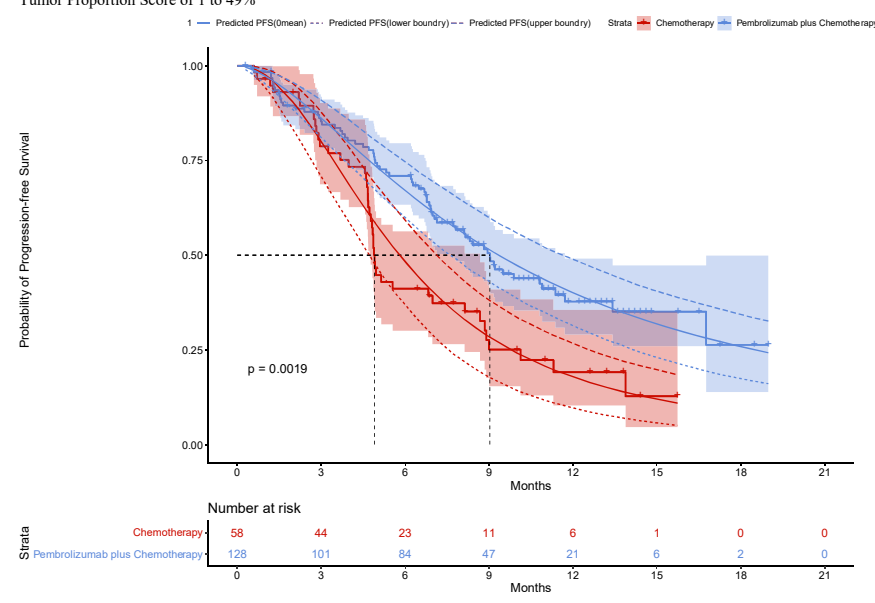

B

Tumor Proportion Score of $<1 \%$
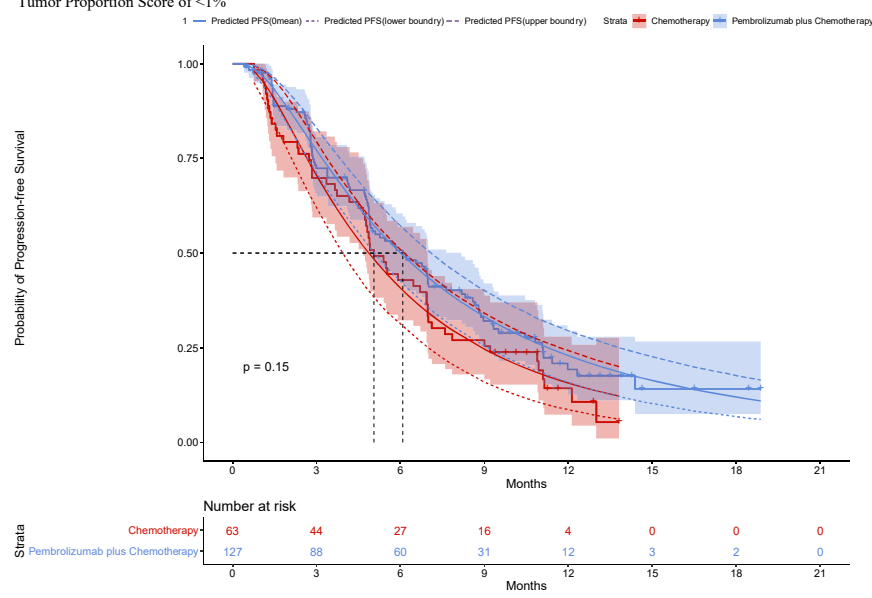

D

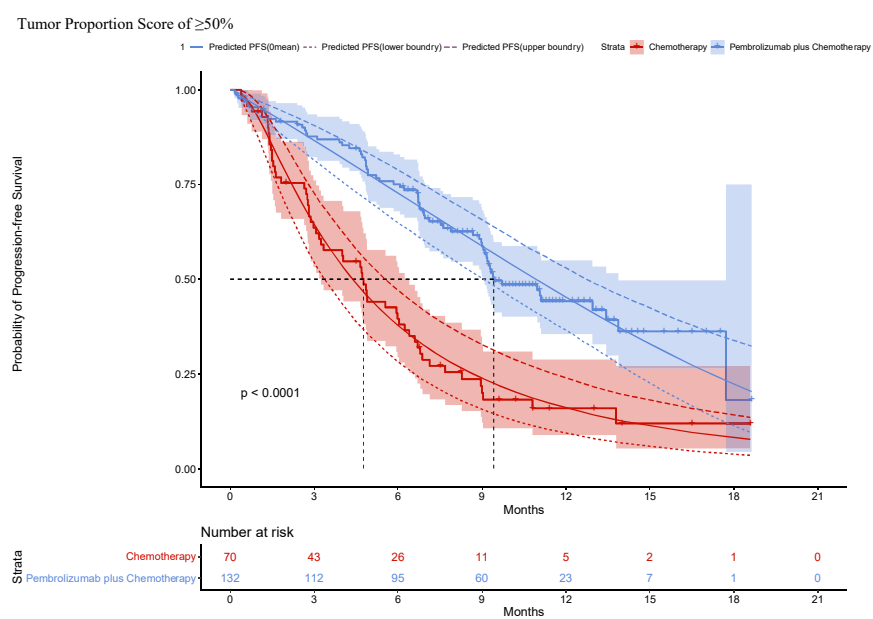

Figure S1 The replicated Kaplan-Meier PFS curves of standard chemotherapy (red) and pembrolizumab plus standard chemotherapy (blue) in KEYNOTE-189 trial. The smooth lines indicated the survival curves predicting their corresponding best survival distributions. The smoothly solid, dashed and dotted lines indicated the mean, upper boundary and lower boundary lines of $95 \%$ CI. PFS, progression-free survival. 
A

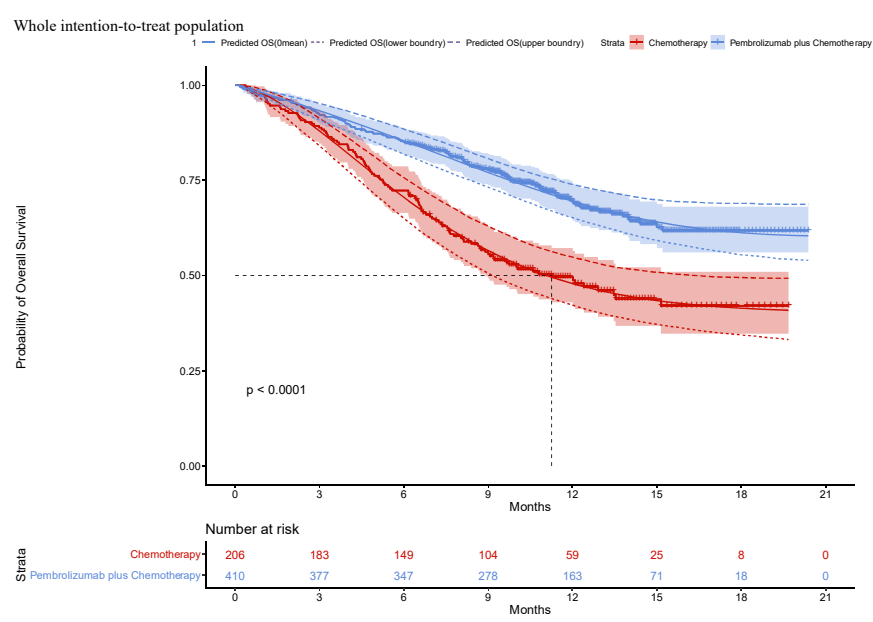

C

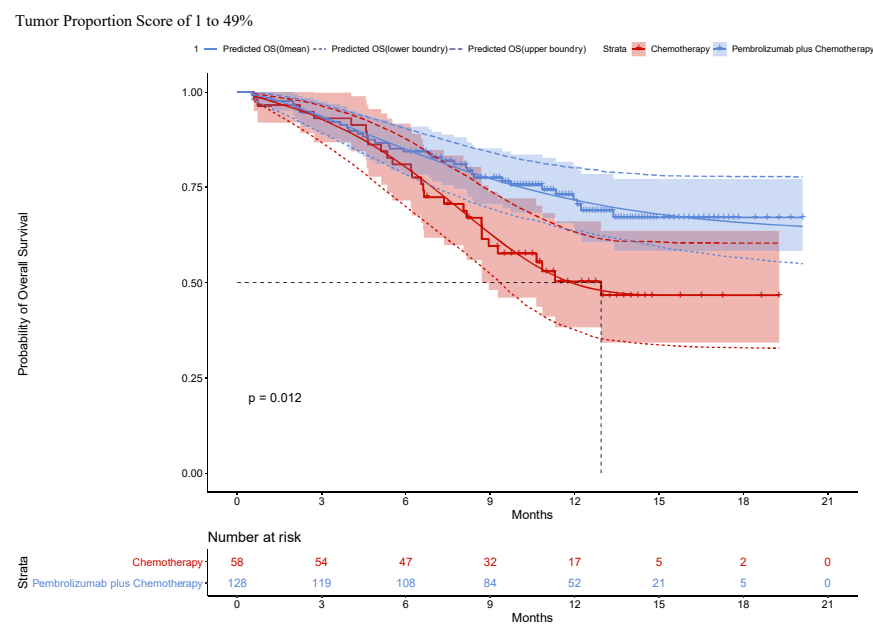

B

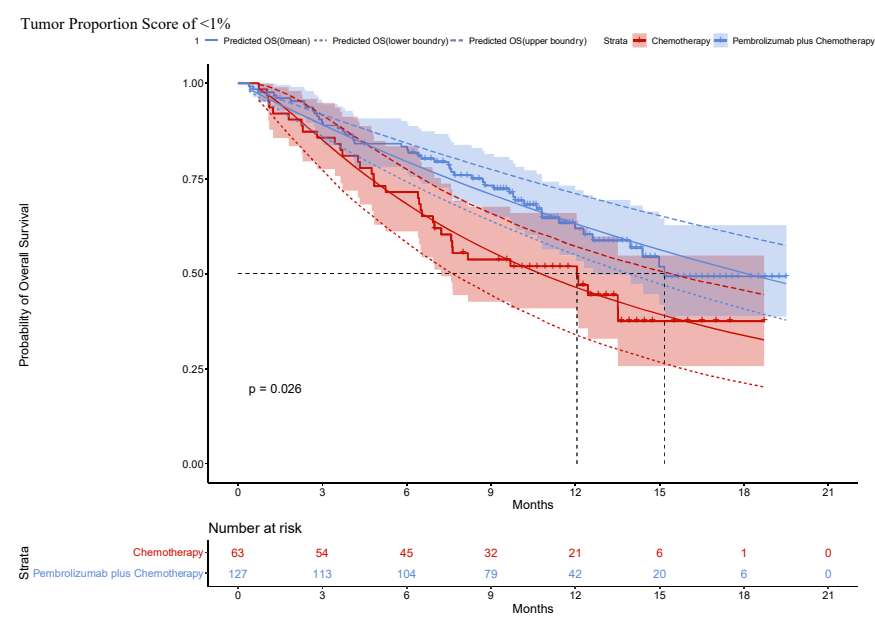

D

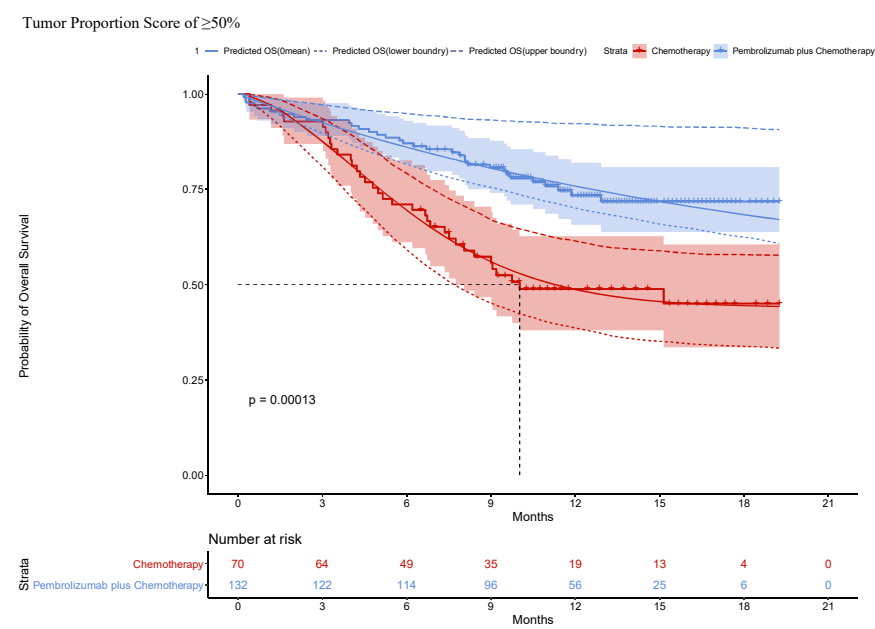

Figure S2 The replicated Kaplan-Meier OS curves of standard chemotherapy (red) and pembrolizumab plus standard chemotherapy (blue) in KEYNOTE-189 trial. The smooth lines indicated the survival curves predicting their corresponding best survival distributions. The smoothly solid, dashed and dotted lines indicated the mean, upper boundary and lower boundary lines of $95 \%$ CI. OS, overall survival. 
A

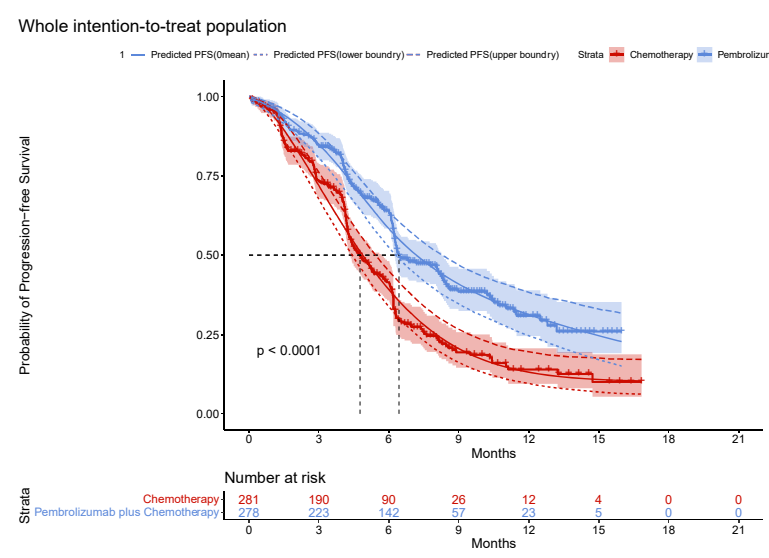

C

Tumor Proportion Score of 1 to $49 \%$

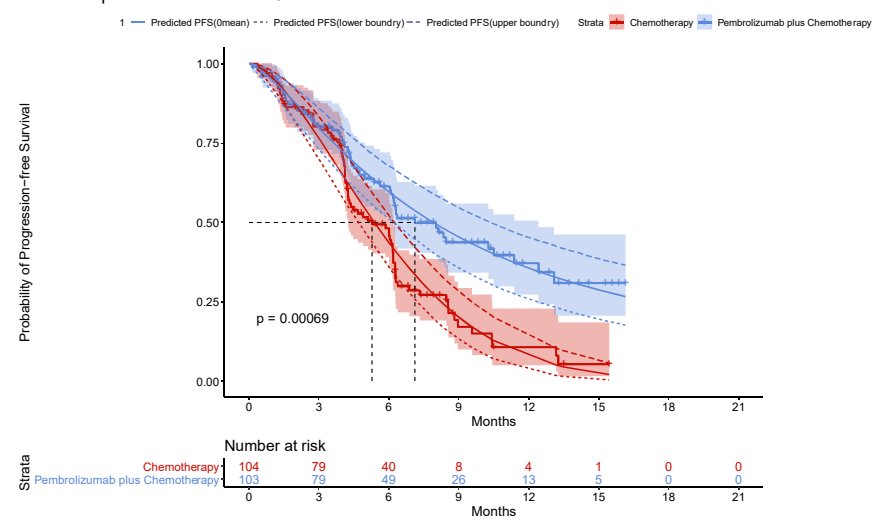

B

Tumor Proportion Score of $<1 \%$

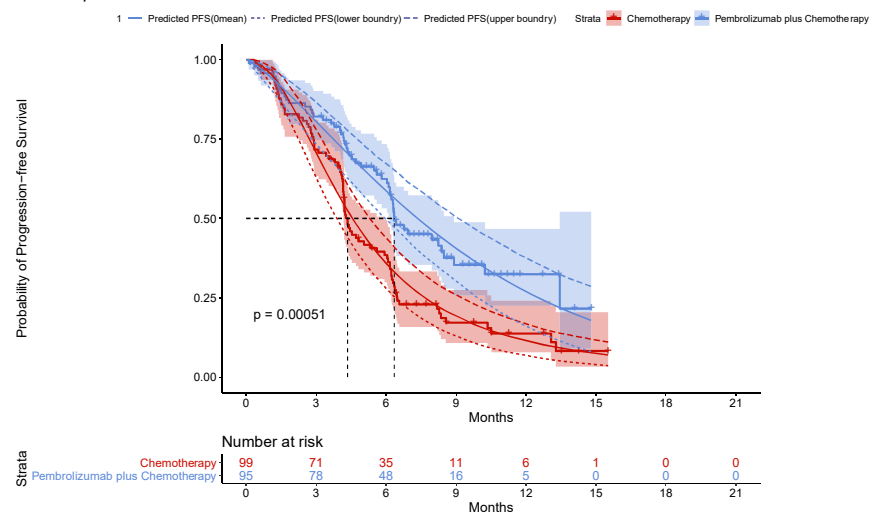

D

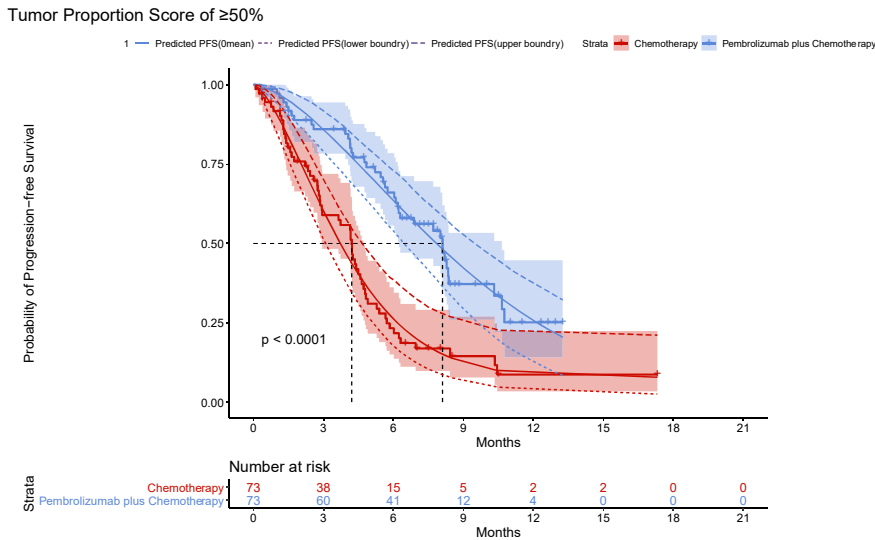

Figure S3 The replicated Kaplan-Meier PFS curves of standard chemotherapy (red) and pembrolizumab plus standard chemotherapy (blue) in KEYNOTE-407 trial. The smooth lines indicated the survival curves predicting their corresponding best survival distributions. The smoothly solid, dashed and dotted lines indicated the mean, upper boundary and lower boundary lines of $95 \%$ CI. PFS, progression-free survival. 
A

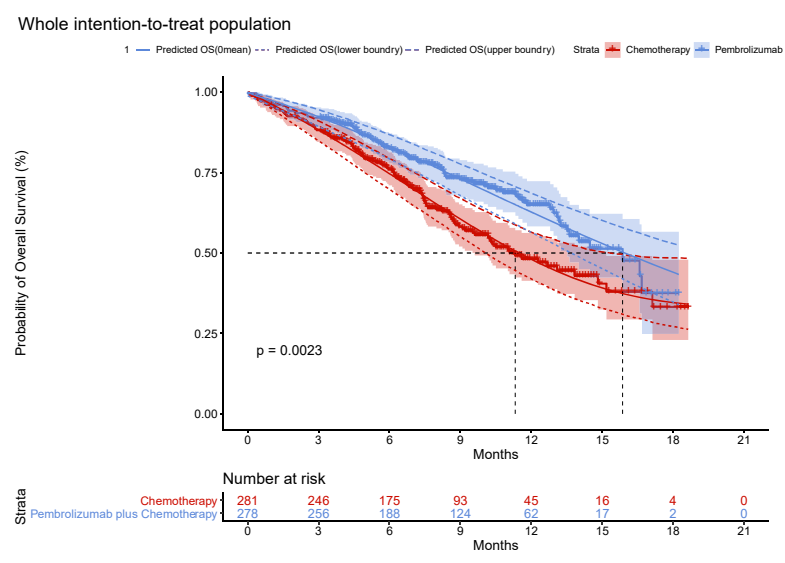

C

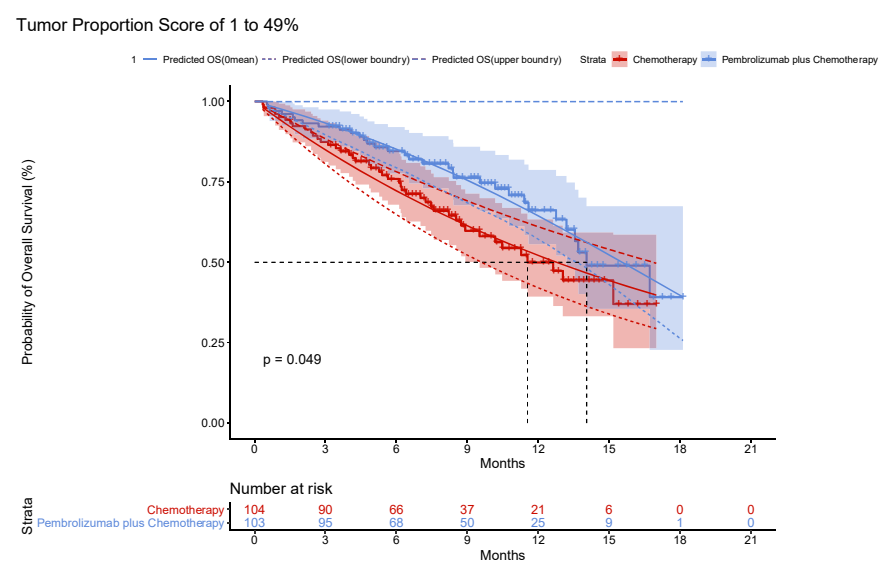

B

Tumor Proportion Score of $<1 \%$

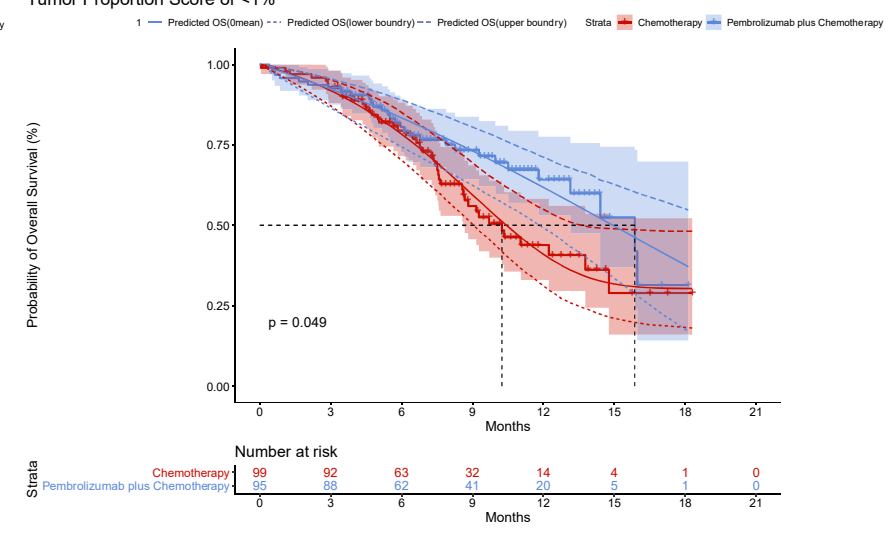

D

Tumor Proportion Score of $\geq 50 \%$

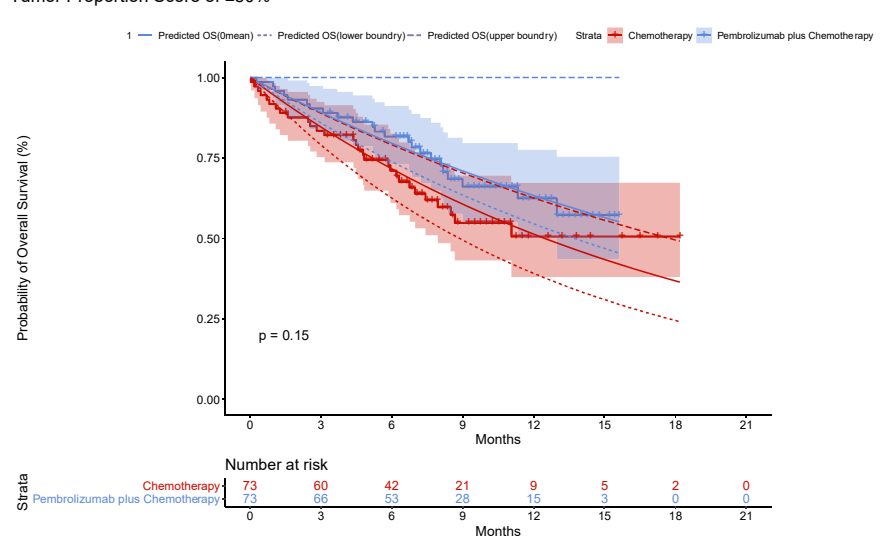

Figure S4 The replicated Kaplan-Meier OS curves of standard chemotherapy (red) and pembrolizumab plus standard chemotherapy (blue) in KEYNOTE-407 trial. The smooth lines indicated the survival curves predicting their corresponding best survival distributions. The smoothly solid, dashed and dotted lines indicated the mean, upper boundary and lower boundary lines of $95 \%$ CI. OS, overall survival. 


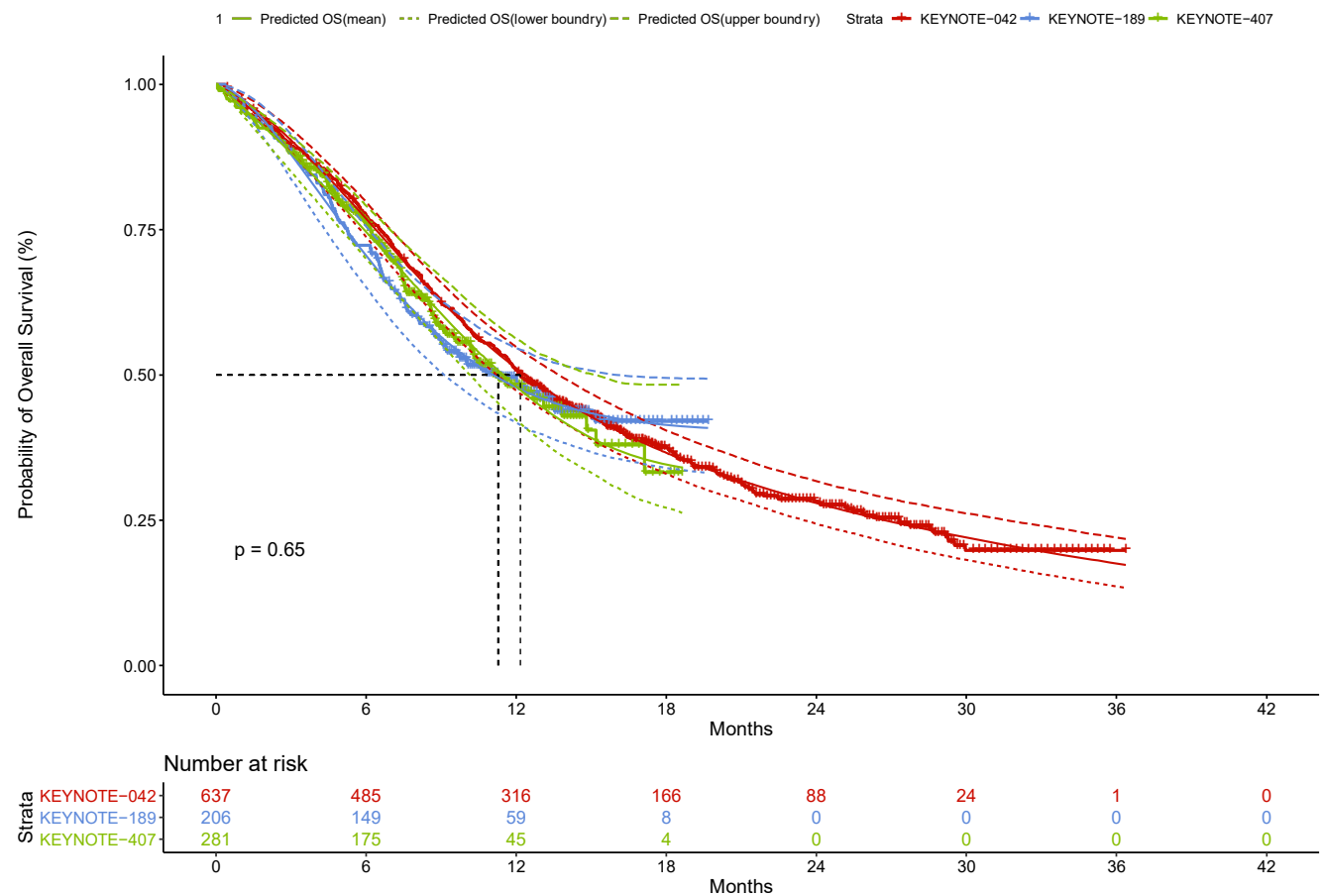

Figure S5 The replicated Kaplan-Meier OS curves of standard chemotherapy in KEYNOTE-042, 189 and 407 trials. The smooth lines indicated the survival curves predicting their corresponding best survival distributions. The smoothly solid, dashed and dotted lines indicated the mean, upper boundary and lower boundary lines of $95 \%$ CI. OS, overall survival. 
Table S2 Hazard ratios of subgroups with confirmed PD-L1 TPS versus whole patients with unconfirmed PD-L1 TPS in chemotherapy arms

\begin{tabular}{|c|c|c|c|c|c|c|}
\hline Trials and populations & \multicolumn{3}{|c|}{ PFS } & \multicolumn{3}{|c|}{ OS } \\
\hline Subgroup with confirmed PD-L1 TPS of $<1 \%$ & 1.29 & 1.07 & 1.56 & 1.22 & 0.95 & 1.57 \\
\hline \multicolumn{7}{|l|}{ KEYNOTE-407 trial } \\
\hline Subgroup with confirmed PD-L1 TPS of $<1 \%$ & 1.09 & 0.89 & 1.33 & 1.05 & 0.81 & 1.37 \\
\hline Subgroup with confirmed PD-L1 TPS of $1 \%$ to $49 \%$ & 0.98 & 0.8 & 1.19 & 0.97 & 0.74 & 1.26 \\
\hline
\end{tabular}

PFS, progression-free survival; OS, overall survival; PD-L1, programmed cell death 1 ligand 1; TPS, tumor proportion score. 
Table S3 Cost estimates in the Chinese setting

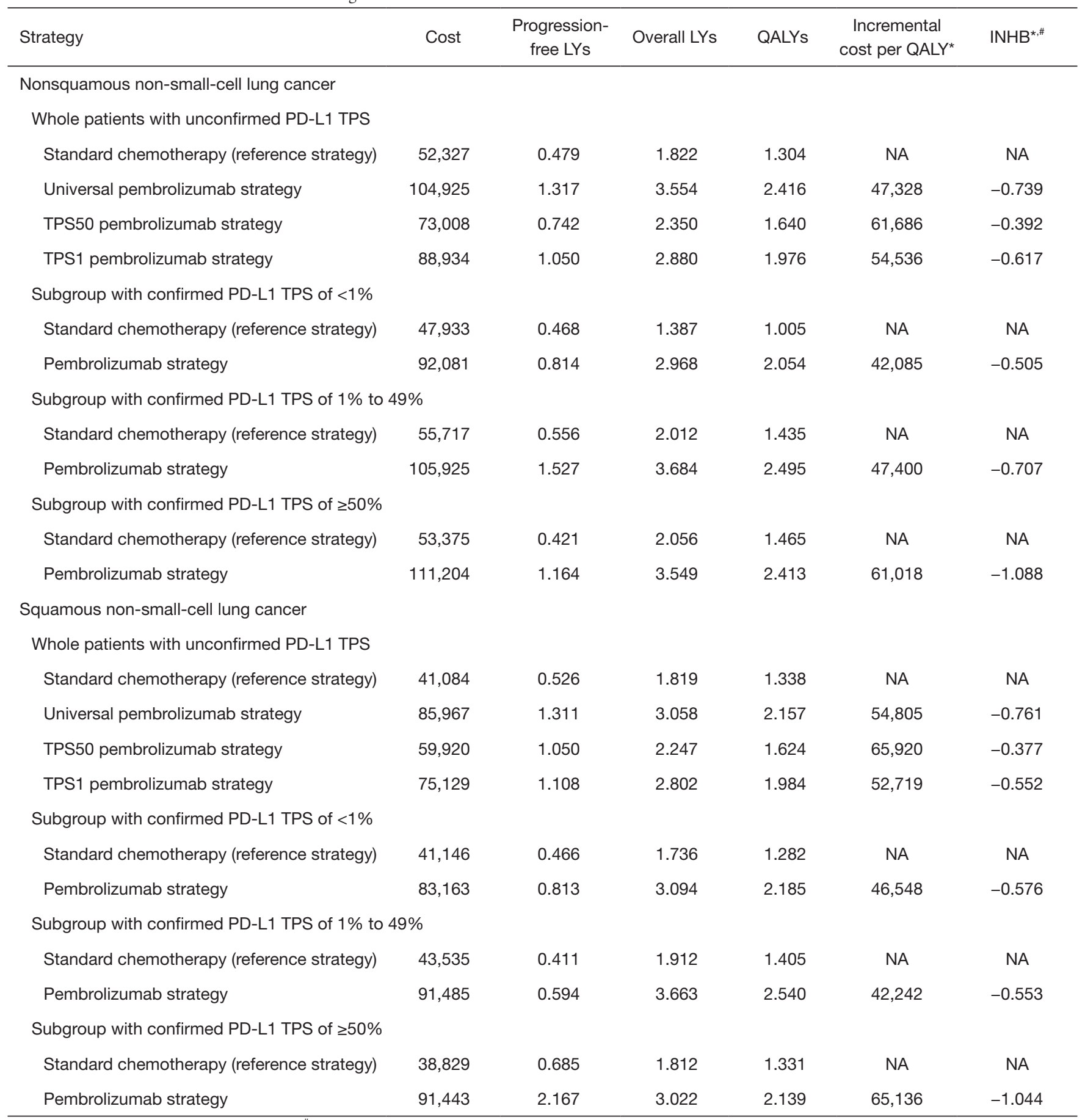

*, comparing with reference strategy; ", three times of Chinese gross domestic product (GDP) per capita in 2018 (\$28,410) was adopted as the willingness-to-pay threshold. QALY, quality-adjusted-life year; INHB, incremental net-health benefit; PD-L1, programmed cell death 1 ligand 1; TPS, tumor proportion score. 
Table S4 Summary of cost (\$) and outcome results in base-case analysis in Chinese setting

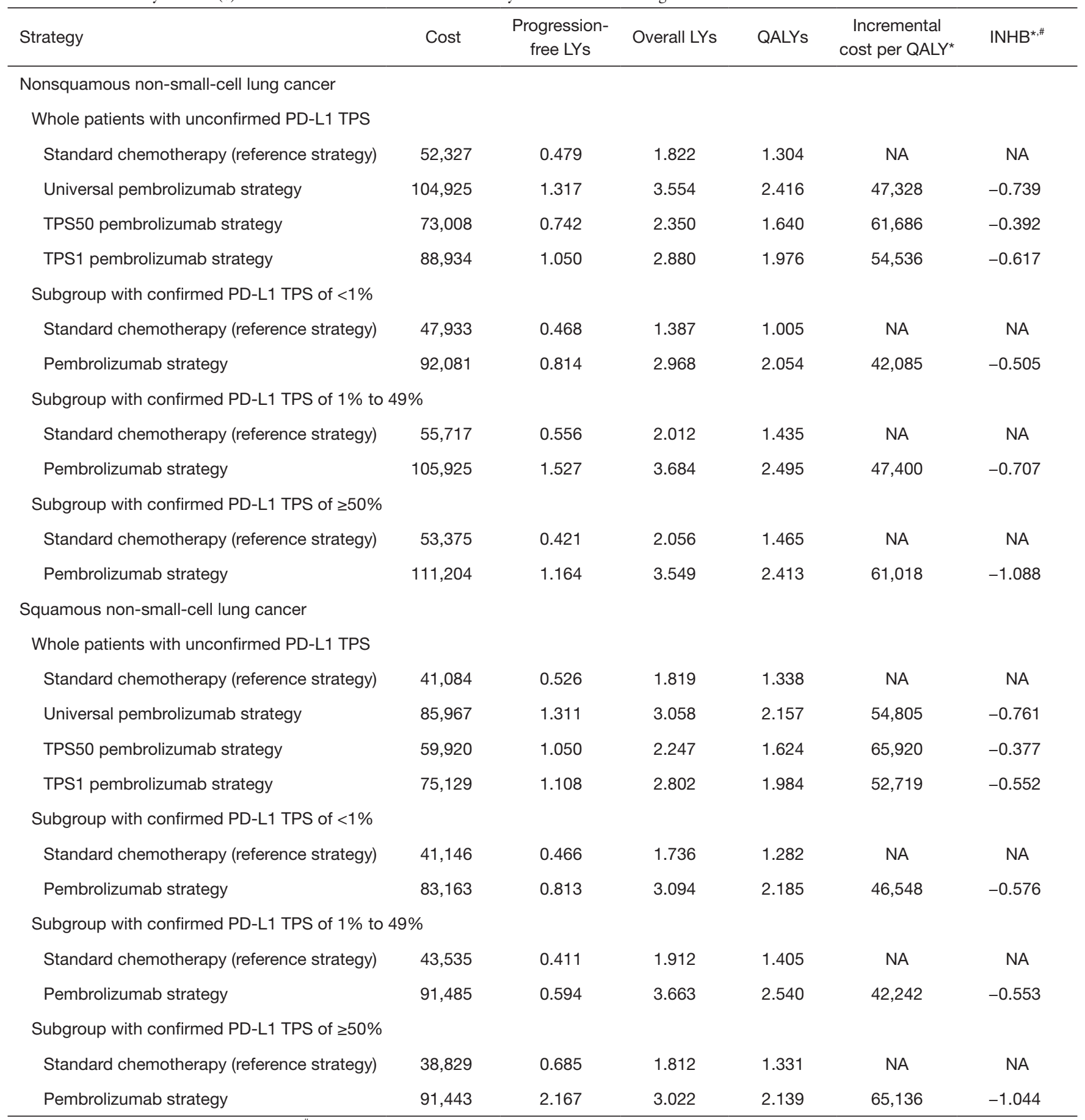

*, comparing with reference strategy; ", three times of Chinese gross domestic product (GDP) per capita in 2018 (\$28,410) was adopted as the willingness-to-pay threshold. QALY, quality-adjusted-life year; INHB, incremental net-health benefit; PD-L1, programmed cell death 1 ligand 1; TPS, tumor proportion score. 